\title{
ON THE PARABOLIC LIPSCHITZ APPROXIMATION OF PARABOLIC UNIFORM RECTIFIABLE SETS
}

\author{
K. NYSTRÖM, M. STRÖMQVIST
}

\begin{abstract}
We prove the existence of big pieces of regular parabolic Lipschitz graphs for a class of parabolic uniform rectifiable sets satisfying what we call a synchronized two cube condition. An application to the fine properties of parabolic measure is given.

2000 Mathematics Subject Classification.

Keywords and phrases: parabolic Lipschitz graph, parabolic uniform rectifiability, big pieces, parabolic measure, caloric measure.
\end{abstract}

\section{INTRODUCTION AND STATEMENT OF MAIN RESULTS}

A by now classical result due to G. David and D. Jerison [DJ] states that if $\Sigma \subset \mathbb{R}^{n}$ is a closed set which is Ahlfors-David regular with respect to the restriction of the (n-1)-dimensional Hausdorff measure to $\Sigma, \sigma$, and if $\Sigma$ satisfies what they call a two disc condition, then $\Sigma$ contains big pieces of Lipschitz graphs in a sense which has to be made precise, see [DJ]. This result has many applications and implications and we here briefly discuss two. First, if $\Omega$ is one component of $\mathbb{R}^{n} \backslash \Sigma$, and if, in addition, $\Omega$ is an NTA-domain in the sense of [JK], then the harmonic measure on $\partial \Omega$ is in the Muckenhoupt class $A_{\infty}$ with respect to $\sigma$. Second, combining [DJ] with the monumental work of G. David and S. Semmes [DS1], see also [DS], one can, if $\Sigma$ is as above, conclude the equivalences between the statements

(i) $\quad \Sigma$ is uniformly rectifiable,

(ii) $\quad \Sigma$ is good for (elliptic) singular integrals,

(iii) $\quad \Sigma$ has a Corona decomposition.

We refer to [DS1] for definitions and precise statements of (1.1) and it is fair to say that [DS1] remains a source of continuous inspiration for anyone interested in the analysis of and on uniformly rectifiable sets.

This paper is the first in a sequence of two papers, motivated by applications to the analysis of parabolic partial differential equations on domains not locally given by graphs, devoted to parabolic versions of the above results. In this paper we prove that if $\Sigma \subset \mathbb{R}^{n+1}$ is a closed set which is Ahlfors-David regular in the parabolic sense, see Definition 1, and if $\Sigma$ satisfies what we call a synchronized two cube condition, see Definition 8 , then $\Sigma$ contains big pieces of Lip (1,1/2) graphs in a sense made precise below. Furthermore, if in addition $\Sigma$ is uniformly rectifiable in the parabolic sense, see Definition 3, then we prove that $\Sigma$ contains big pieces of regular parabolic Lipschitz graphs, graphs which are slightly more regular compared to Lip $(1,1 / 2)$ graphs and which have turned out to be the appropriate graphs from the point of view of parabolic singular integrals and parabolic measure. Building on our results concerning big pieces we in this paper also give an implication for parabolic measure, defined in a component of $\mathbb{R}^{n} \backslash \Sigma$. In the subsequent paper, see [NS], the goal is to establish, if $\Sigma$ is as above, the equivalences between the 
statements

$\left(i^{\prime}\right) \quad \Sigma$ is uniformly rectifiable in the parabolic sense,

$\left(i i^{\prime}\right) \quad \Sigma$ is good for (parabolic) singular integrals,

$\left(i i i^{\prime}\right) \quad \Sigma$ has a parabolic Corona decomposition.

In general the theory of parabolic boundary value problems, and the analysis of and on parabolic uniformly rectifiable sets, is less developed compared to the elliptic counterparts. The main results in the field are due to Hofmann, Lewis, Murray, Silver, see [H], [HL], [HL1], [LM], [LS] and Hofmann, Lewis, Nyström, see [HLN], [HLN1]. In particular, in [H], [HL], [LM], [LS], the authors establish the correct notion of (time-dependent) regular parabolic Lipschitz graphs from the point of view of parabolic singular integrals and parabolic measure. In [HLN], [HLN1] the first author, together with Hofmann and Lewis, introduced the correct notion of parabolic uniformly rectifiable sets and proved the existence of big pieces of regular parabolic Lipschitz graphs under the additional assumption that $\Sigma$ is Reifenberg flat in the parabolic sense. Apart from these results the field is essentially open and as such we claim that this paper and our subsequent paper [NS] advance the field substantially. In general we believe that our results will pave the way for further developments of boundary and free boundary problems for parabolic partial differential equations on domains not locally given by graphs. The ambition is to pursue this line of research in subsequent papers.

1.1. Notation. Points in Euclidean $(n+1)$-space $\mathbb{R}^{n+1}$ are denoted by $(X, t)=$ $\left(x_{1}, \ldots, x_{n}, t\right)$, where $X=\left(x_{1}, \ldots, x_{n}\right) \in \mathbb{R}^{n}$ and $t$ represents the time-coordinate. We will always assume that $n \geq 1$. We let $\bar{E}, \partial E$, be the closure and boundary of the set $E \subset \mathbb{R}^{n+1} \cdot\langle\cdot, \cdot\rangle$ denotes the standard inner product on $\mathbb{R}^{n}$ and we let $|X|=\langle X, X\rangle^{1 / 2}$ be the Euclidean norm of $X$. We let $\|(X, t)\|:=|X|+|t|^{1 / 2}$. Given $(X, t),(Y, s) \in \mathbb{R}^{n+1}$ we let $d_{p}(X, t, Y, s)=|X-Y|+|t-s|^{1 / 2}$ and we define $d(X, t, E)$ to equal the parabolic distance, defined with respect to $d_{p}(\cdot, \cdot)$, from $(X, t) \in \mathbb{R}^{n+1}$ to $E$. We let

$$
Q_{r}(X, t)=\left\{(Y, s) \in \mathbb{R}^{n+1}:\left|y_{i}-x_{i}\right|<r,|t-s|<r^{2}\right\},
$$

whenever $(X, t) \in \mathbb{R}^{n+1}, r>0$, and we call $Q_{r}(X, t)$ a parabolic cube of size $r$. We let $d x$ denote Lebesgue $n$-measure on $\mathbb{R}^{n}$ and we let, given an integer $k \geq 1$, $\mathcal{H}^{k}$ denote the $k$-dimensional Hausdorff measure. Given a Borel set $E \subset \mathbb{R}^{n+1}$ we let $\sigma(E)=\int_{E} d \sigma_{t} d t$ where $d \sigma_{t}$ denotes the restriction of the $(n-1)$-dimensional Hausdorff measure to the time slice $E \cap\left(\mathbb{R}^{n} \times\{t\}\right) . \Sigma \subset \mathbb{R}^{n+1}$ will denote a closed set. Below we will, for $(X, t) \in \Sigma$ and $r>0$, use the notation $\Delta(X, t, r)=\Sigma \cap Q_{r}(X, t)$. The extremal time coordinates of $\Sigma$ will be denoted by $T_{0}=\inf \{t: \exists(X, t) \in \Sigma\}$ and $T_{1}=\sup \{t: \exists(X, t) \in \Sigma\}$. When we consider a domain $\Omega$ defined as a connected component of $\mathbb{R}^{n+1} \backslash \Sigma, T_{0}$ and $T_{1}$ will be defined relative to $\partial \Omega$.

1.2. Regular parabolic graphs. A function $\psi: \mathbb{R}^{n-1} \times \mathbb{R} \rightarrow \mathbb{R}$ is called Lip $(1,1 / 2)$, or Lip $(1,1 / 2)$ regular, with constant $M$, if

$$
|\psi(x, t)-\psi(y, s)| \leq M\left(|x-y|+|t-s|^{1 / 2}\right)
$$

whenever $(x, t) \in \mathbb{R}^{n},(y, s) \in \mathbb{R}^{n}$. We call $\Omega \subset \mathbb{R}^{n+1}$ a (unbounded) Lip $(1,1 / 2)$ graph domain, with constant $M$, if

$$
\Omega=\Omega_{\psi}=\left\{\left(x, x_{n}, t\right) \in \mathbb{R}^{n-1} \times \mathbb{R} \times \mathbb{R}: x_{n}>\psi(x, t)\right\}
$$

for some $\operatorname{Lip}(1,1 / 2)$ function $\psi$ having Lip $(1,1 / 2)$ constant bounded by $M$. We say that $\psi=\psi(x, t): \mathbb{R}^{n-1} \times \mathbb{R} \rightarrow \mathbb{R}$ is a regular parabolic Lip $(1,1 / 2)$ function 
with parameters $b_{1}$ and $b_{2}$, if $\psi$ has compact support and satisfies

(i) $|\psi(x, t)-\psi(y, t)| \leq b_{1}|x-y|, x, y \in \mathbb{R}^{n-1}, t \in \mathbb{R}$,

(ii) $\quad D_{1 / 2}^{t} \psi \in B M O\left(\mathbb{R}^{n}\right), \quad\left\|D_{1 / 2}^{t} \psi\right\|_{*} \leq b_{2}<\infty$.

Here $D_{1 / 2}^{t} \psi(x, t)$ denotes the $1 / 2$ derivative in $t$ of $\psi(x, \cdot), x$ fixed. This half derivative in time can be defined by way of the Fourier transform or by

$$
D_{1 / 2}^{t} \psi(x, t) \equiv \hat{c} \int_{\mathbb{R}} \frac{\psi(x, s)-\psi(x, t)}{|s-t|^{3 / 2}} d s
$$

for properly chosen $\hat{c}$. $\|\cdot\|_{*}$ denotes the norm in parabolic $B M O\left(\mathbb{R}^{n}\right)$. For a definition of the space $B M O\left(\mathbb{R}^{n}\right)$ we refer to [HLN]. It is well known, see [HL], that if $\psi$ is a regular parabolic Lip $(1,1 / 2)$ function with parameters $b_{1}$ and $b_{2}$, then $\psi$ is $\operatorname{Lip}(1,1 / 2)$ regular with constant $M=M\left(b_{1}, b_{2}\right)$. However, there are examples of functions $\psi$ which are Lip $(1,1 / 2)$ regular but not regular parabolic Lip $(1,1 / 2)$, see $[\mathrm{LS}]$. We call $\Omega \subset \mathbb{R}^{n+1}$ a (unbounded) regular parabolic Lip $(1,1 / 2)$ graph domain, with constants $\left(b_{1}, b_{2}\right)$, if (1.4) holds for some regular parabolic Lip $(1,1 / 2)$ function $\psi$ having constants $\left(b_{1}, b_{2}\right)$.

\subsection{Parabolic uniform rectifiability.}

Definition 1. Let $\Sigma \subset \mathbb{R}^{n+1}$ be a closed set. We say that $\Sigma$ is parabolic AhlforsDavid regular, parabolic ADR for short, with constant $M, M \geq 1$, if

$$
M^{-1} r^{n+1} \leq \sigma(\Delta(X, t, r)) \leq M r^{n+1},
$$

whenever $0<r<\operatorname{diam} \Sigma,(X, t) \in \Sigma, T_{0}<t<T_{1}$ and where $\operatorname{diam} \Sigma$ is the (parabolic) diameter of $\Sigma$ (which may be infinite).

Definition 2. Assume that $\Sigma \subset \mathbb{R}^{n+1}$ is parabolic ADR in the sense of Definition 1. Let

$$
\gamma(Z, \tau, r)=\inf _{P}\left(r^{-n-1} \int_{\Delta(Z, \tau, r)}\left(\frac{d(Y, s, P)}{r}\right)^{2} d \sigma(Y, s)\right)^{1 / 2}
$$

whenever $(Z, \tau) \in \Sigma, r>0$, and where the infimum is taken with respect to all $n$ dimensional planes $P$ containing a line parallel to the $t$ axis. Let

$$
d \nu(Z, \tau, r)=\gamma(Z, \tau, r) d \sigma(Z, \tau) r^{-1} d r
$$

We say that $\nu$ is a Carleson measure on $\Delta(Y, s, R) \times(0, R)$ if there exists $\tilde{M}<\infty$ such that

$$
\nu(\Delta(X, t, \rho) \times(0, \rho)) \leq \tilde{M} \rho^{n+1}
$$

whenever $(X, t) \in \Sigma$ and $Q_{\rho}(X, t) \subset Q_{R}(Y, s)$. The least such $\tilde{M}$ in (1.9) is called the Carleson norm of $\Delta(Y, s, R) \times(0, R)$.

Definition 3. Assume that $\Sigma \subset \mathbb{R}^{n+1}$ is parabolic ADR in the sense of Definition 1 with constant $M$. Let $\nu$ be defined as in (4.1). Then $\Sigma$ is parabolic Uniformly Rectifiable, parabolic UR for short, with UR constants $(M, \tilde{M})$ if

$$
\|\nu\|_{+}:=\sup _{(X, t) \in \Sigma, \rho>0} \rho^{-n-1} \nu(\Delta(X, t, \rho) \times(0, \rho)) \leq \tilde{M} .
$$


1.4. Uniform big pieces. Assume that $\Sigma \subset \mathbb{R}^{n+1}$ is parabolic ADR in the sense of Definition 1. Let in the following $\pi$ denote the orthogonal projection onto the plane $\left\{\left(x, x_{n}, t\right) \in \mathbb{R}^{n-1} \times \mathbb{R} \times \mathbb{R}: x_{n}=0\right\}$. At instances we identify $\mathbb{R}^{n}$ with $\mathbb{R}^{n-1} \times\{0\} \times \mathbb{R}$, and put

$$
I_{r}(z, \tau)=\left\{(y, s) \in \mathbb{R}^{n}:\left|y_{i}-z_{i}\right|<r, i=1, \ldots, n-1,|s-\tau|<r^{2}\right\}
$$

for $(z, \tau) \in \mathbb{R}^{n}, r>0$.

Definition 4. We say that $\Sigma$ contains uniform big pieces of Lip $(1,1 / 2)$ graphs with constants $(\epsilon, \hat{M})$ if the following hold. Given $(X, t) \in \Sigma, T_{0}<t<T_{1}$ and $0<R<\operatorname{diam} \Sigma$, there exists, after a possible rotation in the space variable, a Lip $(1,1 / 2)$ function $\psi$ with constant $\hat{M}$, and $\epsilon>0$, such that

$$
\mathcal{H}^{n}\left(\pi\left(\Sigma_{\psi} \cap \Delta(X, t, R)\right)\right) \geq \epsilon R^{n+1},
$$

where

$$
\Sigma_{\psi}=\left\{\left(x, x_{n}, t\right) \in \mathbb{R}^{n-1} \times \mathbb{R} \times \mathbb{R}: x_{n}=\psi(x, t)\right\} .
$$

Definition 5. We say that $\Sigma$ contains uniform big pieces of regular parabolic Lip $(1,1 / 2)$ graphs with constants $\left(\epsilon, b_{1}, b_{2}\right)$ if, whenever $(X, t) \in \Sigma, T_{0}<t<T_{1}$ and $0<R<\operatorname{diam} \Sigma,(1.11)$ and (1.12) hold with a regular parabolic Lip $(1,1 / 2)$ function $\psi$, satisfying (1.5) with constants $b_{1}, b_{2}$, and for $\epsilon>0$.

Definition 6. Let $\Omega \subset \mathbb{R}^{n+1}$ be a domain, i.e., a connected open set, defined as a connected component of $\mathbb{R}^{n+1} \backslash \Sigma$. We then say that $\partial \Omega$ satisfies a uniform interior big pieces of $\operatorname{Lip}(1,1 / 2)$ graphs condition with constants $(\epsilon, \hat{M}, C)$ if the following hold. Given $(\hat{X}, \hat{t}) \in \Omega$, we can find a domain $\tilde{\Omega}$ and $C \geq 1$ such that the following holds with $d:=d(\hat{X}, \hat{t}, \partial \Omega)$.

(i) $\quad \tilde{\Omega} \subset \Omega \cap Q_{C d}(\hat{X}, \hat{t})$.

(ii) After a possible rotation in the space variables we have

$$
\tilde{\Omega}=\left\{\left(y, y_{n}, s\right):(y, s) \in I_{\hat{M}^{-1} d}(\hat{x}, \hat{t}), \psi(y, s)<y_{n}<\hat{x}_{n}+d / 2\right\},
$$
where $(\hat{X}, \hat{t})=\left(\hat{x}, \hat{x}_{n}, \hat{t}\right)$ and for some Lip $(1,1 / 2)$ function $\psi$ with constant $\hat{M}$.

(1.13)(iii) $\quad \mathcal{H}^{n}\left(\pi(\partial \Omega \cap \partial \tilde{\Omega}) \cap I_{\hat{M}^{-1} d}(\hat{x}, \hat{t})\right) \geq \epsilon d^{n+1}$.

Definition 7. Let $\Omega \subset \mathbb{R}^{n+1}$ be a domain, i.e., a connected open set, defined as a connected component of $\mathbb{R}^{n+1} \backslash \Sigma$. We then say that $\partial \Omega$ satisfies a uniform interior big pieces of regular parabolic Lip $(1,1 / 2)$ graphs condition with constants $\left(\epsilon, b_{1}, b_{2}, C\right)$ if the following hold. Given $(\hat{X}, \hat{t}) \in \Omega$, we can find a domain $\tilde{\Omega}$ such that $(i)-($ iii $)$ of Definition 6 hold for some regular parabolic Lip $(1,1 / 2)$ function $\psi$ with constants $\left(b_{1}, b_{2}\right)$ and for some constant $\hat{M}=\hat{M}\left(b_{1}, b_{2}\right)$.

Remark 1.1. Note that (1.11) implies, as Hausdorff measure does not increase under projections, that

$$
\sigma\left(\Sigma_{\psi} \cap \Delta(X, t, R)\right) \geq \epsilon R^{n+1} .
$$

\subsection{Statement of main results - existence of big pieces.}

Definition 8. Assume that $\Sigma \subset \mathbb{R}^{n+1}$ is parabolic ADR in the sense of Definition 1 with constant $M$. Let $\gamma_{0} \geq 1$ be given. We say that $\Sigma$ satisfies a synchronized two cube condition with constant $\gamma_{0}$, if there exist, for all $(X, t) \in \Sigma, T_{0}<t<$ $T_{1}$ and $0<r<\operatorname{diam} \Sigma$, two parabolic cubes $Q_{\rho}\left(X_{1}, t_{1}\right), Q_{\rho}\left(X_{2}, t_{2}\right)$, such that $Q_{\rho}\left(X_{1}, t_{1}\right) \cap\left(\mathbb{R}^{n} \times\left(T_{0}, T_{1}\right)\right)$ and $Q_{\rho}\left(X_{1}, t_{1}\right) \cap\left(\mathbb{R}^{n} \times\left(T_{0}, T_{1}\right)\right)$ belong to different connected components of $\mathbb{R}^{n+1} \backslash \Sigma$, with

$$
\gamma_{0}^{-1} r<\rho<r, t_{1}=t=t_{2},\left\|(X, t)-\left(X_{1}, t\right)\right\| \leq r,\left\|(X, t)-\left(X_{2}, t\right)\right\| \leq r .
$$


Definition 9. Assume that $\Sigma \subset \mathbb{R}^{n+1}$ is parabolic ADR in the sense of Definition 1 with constant $M$. Let $\gamma_{0} \geq 1$ be given. Let $\Omega \subset \mathbb{R}^{n+1}$ be a domain defined as a connected component of $\mathbb{R}^{n+1} \backslash \Sigma$. We say that $\partial \Omega$ satisfies a synchronized two cube condition with constant $\gamma_{0}$, if there exist, for all $(X, t) \in \partial \Omega, T_{0}<t<$ $T_{1}$ and $0<r<\operatorname{diam} \Sigma$, two parabolic cubes $Q_{\rho}\left(X_{1}, t_{1}\right), Q_{\rho}\left(X_{2}, t_{2}\right)$, such that $Q_{\rho}\left(X_{1}, t_{1}\right) \cap\left(\mathbb{R}^{n} \times\left(T_{0}, T_{1}\right)\right) \subset \Omega, Q_{\rho}\left(X_{2}, t_{2}\right) \cap\left(\mathbb{R}^{n} \times\left(T_{0}, T_{1}\right)\right) \subset \mathbb{R}^{n+1} \backslash \bar{\Omega}$, satisfying

$$
\gamma_{0}^{-1} r<\rho<r, t_{1}=t=t_{2},\left\|(X, t)-\left(X_{1}, t\right)\right\| \leq r,\left\|(X, t)-\left(X_{2}, t\right)\right\| \leq r .
$$

Remark 1.2. We use the term a synchronized two cube condition to emphasize that if $(X, t) \in \Sigma$, then the $t$-coordinates of the centers of the cylinders $Q_{\rho}\left(X_{1}, t_{1}\right)$, $Q_{\rho}\left(X_{2}, t_{2}\right)$ coincide with $t$.

Concerning big pieces, the following two theorems are our main results.

Theorem 1.1. Let $\Sigma$ be a closed subset of $\mathbb{R}^{n+1}$ which is parabolic ADR with constant $M$. Assume that $\Sigma$ satisfies a synchronized two cube condition with constant $\gamma_{0} \geq 1$, in the sense of Definition 8. Then $\Sigma$ contains uniform big pieces of Lip (1,1/2) graphs with constants $(\epsilon, \hat{M})$ for some $\hat{M}=\hat{M}\left(n, M, \gamma_{0}\right)$, and $\epsilon=\epsilon\left(n, M, \gamma_{0}\right)$. Let $\Omega \subset \mathbb{R}^{n+1}$ be a connected component of $\mathbb{R}^{n+1} \backslash \Sigma$. Assume that $\partial \Omega$ satisfies a synchronized two cube condition with constant $\gamma_{0} \geq 1$ in the sense of Definition 9. Then $\partial \Omega$ satisfies a uniform interior big pieces of Lip (1,1/2) graphs conditions with constants $(\epsilon, \hat{M}, C)$ for some $\epsilon=\epsilon\left(n, M, \gamma_{0}\right), \hat{M}=\hat{M}\left(n, M, \gamma_{0}\right)$, and $C=C\left(n, M, \gamma_{0}\right)$.

Theorem 1.2. Let $\Sigma$ be a closed subset of $\mathbb{R}^{n+1}$ which is parabolic ADR with constant $M$. Assume that $\Sigma$ satisfies a synchronized two cube condition with constant $\gamma_{0} \geq 1$ in the sense of Definition 8. Assume in addition that $\Sigma$ is UR with constants $(M, \tilde{M})$. Then $\Sigma$ contains uniform big pieces of regular parabolic Lip (1,1/2) graphs with constants $\left(\epsilon, b_{1}, b_{2}\right)$ for some $b_{1}=b_{1}\left(n, M, \tilde{M}, \gamma_{0}\right), b_{2}=b_{2}\left(n, M, \tilde{M}, \gamma_{0}\right)$ and $\epsilon=\epsilon\left(n, M, \tilde{M}, \gamma_{0}\right)$. Let $\Omega \subset \mathbb{R}^{n+1}$ be a connected component of $\mathbb{R}^{n+1} \backslash \Sigma$. Assume that $\partial \Omega$ satisfies a synchronized two cube condition with constant $\gamma_{0} \geq 1$ in the sense of Definition 9. Then $\partial \Omega$ satisfies a uniform interior big pieces of regular parabolic Lip (1,1/2) graphs conditions with constants $\left(\epsilon, b_{1}, b_{2}, C\right)$ for some $\epsilon=\epsilon\left(n, M, \gamma_{0}\right)$, $b_{1}=b_{1}\left(n, M, \tilde{M}, \gamma_{0}\right), b_{2}=b_{2}\left(n, M, \tilde{M}, \gamma_{0}\right)$ and $C=C\left(n, M, \gamma_{0}\right)$.

Remark 1.3. Note that Theorem 1.1 is a parabolic version of the main result in [DJ]. In Theorem 1 in [HLN] the conclusion of Theorem 1.2 was established assuming that $\Sigma$ is parabolic ADR with constant $M$, parabolic UR and, in addition, that $\Sigma$ has the separation property and is $\delta$-Reifenberg flat for a sufficiently small $\delta$. If $\Sigma$ has the separation property and is $\delta$-Reifenberg flat, then $\Sigma$ satisfies a synchronized two cube condition. This implication can not be reversed. Hence Theorem 1.2 generalizes Theorem 1 in [HLN] beyond the hypothesis of $\Sigma$ being Reifenberg flat.

Remark 1.4. In [RN1]-[RN3] the author claims several of the results that we establish in this paper and in [NS]. However, in [RN1]-[RN3] the author, unfortunately, in general either gives no proofs of his claims or he supplies proofs which have obvious gaps. Here and in $[\mathrm{NS}]$ we give complete proofs of our results.

1.6. Proofs and organization of the paper. The proof of Theorem 1.1 is given in Section 2. We prove Theorem 1.1 by adapting to the parabolic setting the original and quite ingenious argument of G. David and D. Jerison [DJ]. In Section 3 we prove Theorem 1.2 and to complete the proof we can, after some preliminaries, reuse important results from [HLN]. In Section 4 we prove further results devoted to connectivity, big pieces and parabolic measure. In particular, in Theorem 4.1 
we prove that connectivity of $\Omega \subset \mathbb{R}^{n+1}$, formulated in terms of a uniform Carleson chain condition, implies the synchronized two cube condition, and hence the existence of big pieces. In the elliptic setting, see [LV1], and [BL], [LV], this type of results have been proved to be useful. In Theorem 4.2, assuming the synchronized two cube condition and the Harnack chain property, see Definition 13 below, we formulate a consequence of our main results to the fine properties of parabolic measure.

\section{The Proof of TheOrem 1.1}

We here prove Theorem 1.1. We will only give the proof in the case when $\operatorname{diam} \Sigma=\infty, T_{0}=-\infty$ and $T_{1}=\infty$. If $T_{0}$ or $T_{1}$ is finite, the proof will be completely analogous but all sets ocurring have to be intersected with $\mathbb{R}^{n} \times\left(T_{0}, T_{1}\right)$ and the notation will be more cumbersome. Let $\Sigma$ be a closed subset of $\mathbb{R}^{n+1}$ which is parabolic ADR with constant $M$. Assume that $\Sigma$ satisfies a synchronized two cube condition with constant $\gamma_{0}$. We redefine

$$
M \text { to equal } \max \left\{M, \gamma_{0}, 4 n\right\} .
$$

Based on this we can in the following, without loss of generality, assume that $\Sigma$ is parabolic ADR with constant $M$ and that there exist, for all $(X, t) \in \Sigma$ and $r>0$, two parabolic cubes $Q_{\rho}\left(X_{1}, t_{1}\right), Q_{\rho}\left(X_{2}, t_{2}\right)$, belonging to different connected components of $\mathbb{R}^{n+1} \backslash \Sigma$, with $t_{1}=t=t_{2},\left\|(X, t)-\left(X_{1}, t\right)\right\| \leq r,\left\|(X, t)-\left(X_{1}, t\right)\right\| \leq$ $r$, and

$$
\rho=M^{-1} r .
$$

Consider $(X, t) \in \Sigma$ and $r>0$. We first note, using (2.2) and as the statements and conclusions of Theorem 1.1 are invariant under parabolic scalings and Euclidean translations, that to prove Theorem 1.1 we can without loss of generality assume that

$$
r=2 M \text { and }(X, t)=(0,0) .
$$

Then, by construction there is a point $(Y, 0)$, with $\|(Y, 0)\| \leq 2 M$, and a cube $Q_{2}(Y, 0)$ contained in a component $\mathcal{U}$ of $\mathbb{R}^{n+1} \backslash \Sigma$. Furthermore, if we let $\rho_{0}=$ $(2(1+M))^{-1}$, then there is a point $(Z, 0)$, with $\|(Z, 0)\| \leq M \rho_{0}$, such that the cube $Q_{\rho_{0}}(Z, 0)$ is contained in a component of $\mathbb{R}^{n+1} \backslash \Sigma$ different from $\mathcal{U}$. Indeed, this follows if we apply the synchronized two cube condition at $(0,0)$ and on scale $M \rho_{0}$. Consider the time-independent hyperplane which passes through $(0,0)$ and which has (spatial) normal defined by $Y$. In the following we can assume, after a possible rotation in the spatial coordinates, if necessary, that any point $(X, t) \in$ $\mathbb{R}^{n+1}$ is given as $(X, t)=\left(x, x_{n}, t\right) \in \mathbb{R}^{n-1} \times \mathbb{R} \times \mathbb{R}$, and that $Y=(0, \bar{M})$, where $2 \leq \bar{M} \leq 2 M$. We identify the hyperplane with $\mathbb{R}^{n-1} \times\{0\} \times \mathbb{R}$, we let $\pi$ denote the orthogonal projection onto this plane and we let $\pi^{\perp}$ denote the orthogonal projection onto the normal to this plane. We let

$$
I_{r}(z, \tau)=\left\{(y, s) \in \mathbb{R}^{n}:\left|y_{i}-z_{i}\right|<r, i=1, \ldots, n-1,|s-\tau|<r^{2}\right\},
$$

whenever $(z, \tau) \in \mathbb{R}^{n}, r>0$, we set $I^{0}=I_{1}(0,0)$ and introduce

$$
I^{\bar{M}}:=\left\{\left(x, x_{n}, t\right):(x, t) \in I^{0}, x_{n}=\bar{M}\right\} .
$$

By construction $I^{\bar{M}} \subset \mathcal{U}, d\left(I^{\bar{M}}, \Sigma\right) \geq 1$ and by the choice $\rho_{0}=(2(1+M))^{-1}$ we see that

and that

$$
D:=\pi\left(Q_{\rho_{0}}(Z, 0)\right) \subset \frac{1}{2} I^{0}
$$

there exists $\gamma>0$, depending only on $n$ and $M$, 
such that

$$
\sigma(D) \geq 2 \gamma
$$

Note that any line in the $x_{n}$-direction connecting $D \times\left\{x_{n}=-M\right\}$ with $I^{\bar{M}}$ has to intersect $Q_{\rho_{0}}(Z, 0)$ and $Q_{2}(Y, 0)$, thus it also intersects $\Sigma$. Given a degree of freedom $h>0$ we let

$$
\Gamma=\Gamma_{h}=\left\{(X, t) \in \mathbb{R}^{n+1}: x_{n} \geq h\|(x, t)\|\right\},
$$

i.e., $\Gamma$ is a parabolic cone with aperture defined by $h$, and we let

$$
\begin{aligned}
S= & \left\{(X, t)=\left(x, x_{n}, t\right) \in \Sigma:-M \leq x_{n} \leq \bar{M} \text { and all }(Y, s) \in(X, t)+\Gamma\right. \\
& \text { with } \left.y_{1}=\bar{M} \text { belong to } I^{\bar{M}}\right\} .
\end{aligned}
$$

Let $\bar{M}$ and $M$ be as above. Below we will construct $h$ subject to

$$
h \geq 3 M \geq \bar{M}+M \text {. }
$$

Then, by construction we see that if $(X, t)=\left(x, x_{n}, t\right) \in \Sigma$, and if $(x, t) \in D$, then any $(Y, s) \in(X, t)+\Gamma$ such that $y_{n}=\bar{M}$, belongs to $I^{\bar{M}}$. In particular, $D \subset \pi(S)$ and thus by $(2.5)$,

$$
\mathcal{H}^{n}(\pi(S)) \geq 2 \gamma .
$$

To prove the first statement in Theorem 1.1 it therefore suffices to prove the following lemma.

Lemma 2.1. Let $\gamma$ be as in (2.4), (2.5). Then there exists $h>0$, depending only on $n$ and $M$, such that if we let $\Gamma=\Gamma_{h}$, and if we define

$$
W:=\left\{(x, t) \in I^{0}: \exists(X, t)=\left(x, x_{n}, t\right) \in S,((X, t)+\Gamma) \cap S=\{(X, t)\}\right\},
$$

then $\mathcal{H}^{n}(\pi(S) \backslash W) \leq \gamma$.

Indeed, using Lemma 2.1 we conclude that there is a $\operatorname{Lip}(1,1 / 2)$ graph $G$ with constant $h$ such that $W=\pi(\Sigma \cap G)$ satisfies $\mathcal{H}^{n}(\pi(S) \backslash W) \leq \gamma$, implying $\mathcal{H}^{n}(W) \geq$ $\gamma$. Thus $\sigma\left(\Sigma \cap G \cap Q_{2 M}(0,0)\right) \geq \gamma$ and the proof of the first statement in Theorem 1.1 is complete. Note that by this we have inferred the existence of a $\operatorname{Lip}(1,1 / 2)$ function $\psi$ such that

$$
G \cap\left\{(X, t) \in G: \pi(X, t) \in I^{0}\right\}=\left\{(x, \psi(x, t), t):(x, t) \in I^{0}\right\} .
$$

Define $\tilde{\Omega}$ to be the set

$$
\tilde{\Omega}=\left\{(X, t):(x, t)=\pi(X, t) \in \operatorname{int} I^{0}, \psi(x, t)<x_{n}<\bar{M}\right\},
$$

where int $I^{0}$ is the interior of $I^{0}$. Then

$$
\tilde{\Omega} \subset \Omega \text {. }
$$

The reason for this is that if $(X, t) \in S$ and $\pi(X, t) \in W$, then

$$
((X, t)+\Gamma) \cap\left\{x_{n} \leq \bar{M}\right\} \backslash\{(X, t)\} \subset \Omega,
$$

and that $((X, t)+\Gamma) \cap\left\{x_{n}=\bar{M}\right\} \subset I^{\bar{M}}$. Furthermore, $\partial \tilde{\Omega}$ is $\operatorname{Lip}(1,1 / 2)$ regular and $\sigma(\partial \tilde{\Omega} \cap \partial \Omega) \geq \gamma$.

To prove the second statement in Theorem 1.1, we have to complement the argument above leading up to $(2.7),(2.8)$. Indeed, consider $(\hat{X}, \hat{t}) \in \Omega$ and let $d(\hat{X}, \hat{t}, \partial \Omega)=d$. We first claim that there exists $C=C(n, M) \geq 1$, and

$$
(X, \hat{t}) \in \partial \Omega \text { such that }\|(X, \hat{t})-(\hat{X}, \hat{t})\| \leq C d \text {. }
$$

To prove (2.9), we use the synchronized two cube condition. Indeed, let $(Z, t) \in$ $\partial \Omega$ be a point that satisfies $\|(Z, t)-(\hat{X}, \hat{t})\|=d$. Then there exist two cubes $Q_{d}\left(X_{1}, t_{1}\right) \subset \mathbb{R}^{n+1} \backslash \Omega$ and $Q_{d}\left(X_{2}, t_{2}\right) \subset \Omega$ such that $t_{1}=t=t_{2}$ and $\|(Z, t)-$ $\left(X_{i}, t_{i}\right) \| \leq M d, i=1,2$. By construction $Q_{d}\left(X_{1}, t_{1}\right)$ contains a point $\left(\hat{X}_{1}, \hat{t}\right)$ that 
belongs to $\mathbb{R}^{n+1} \backslash \Omega$. Thus the line connecting $(\hat{X}, \hat{t})$ and $\left(\hat{X}_{1}, \hat{t}\right)$ intersects $\partial \Omega$ at a point $(X, \hat{t})$. It is easy to see that $\|(X, \hat{t})-(\hat{X}, \hat{t})\| \leq(M+\sqrt{n}+2) d$. This proves (2.9) with $C=M+\sqrt{n}+2$. We will now use (2.9) to prove that $\partial \Omega$ satisfies a uniform interior big pieces of $\operatorname{Lip}(1,1 / 2)$ graphs condition in the sense of Definition 6. To start the argument, we note that it follows, as $d(\hat{X}, \hat{t}, \partial \Omega)=d$ and $M \geq 1$, that the cube $Q_{c(n) d / M}(\hat{X}, \hat{t})$ is contained in $\Omega$ if $c(n) \leq(\sqrt{n}+1)^{-1}$. By a parabolic scaling and an Euclidean translation we can in the following, without loss of generality, assume that $(X, \hat{t})=(0,0)$ and $C d=2 M$, where $(X, \hat{t})$ and $C$ are as in $(2.9)$. By this change of coordinates we see that $(\hat{X}, \hat{t})$ and $Q_{c(n) d / M}(\hat{X}, \hat{t})$ are transformed into $(\hat{Y}, 0)$ and $Q_{2 c(n) / C}(\hat{Y}, 0)$, respectively. From this point the argument proceeding Lemma 2.1 can be carried out with $Q_{2 c(n) / C}(\hat{Y}, 0)$ in place of $Q_{2}(Y, 0)$. Furthermore, we must replace $Q_{\rho_{0}}$ and $I^{0}$ by the corresponding sets rescaled by the factor $c(n) / C$. Doing this we obtain, after a rotation of the spacial variables if necessary, and as in (2.7), (2.8), the interior domain

$$
\tilde{\Omega}=\left\{(X, t):(x, t) \in \operatorname{int} I_{c(n) / C}(0,0), \psi(x, t)<x_{n}<\bar{M}\right\} .
$$

By translating and scaling back, but not rotating, to the original coordinates we can conclude that

$$
\tilde{\Omega}=\left\{\left(y, y_{n}, s\right):(y, s) \in \operatorname{int} I_{c(n) d /(2 M)}(\hat{x}, \hat{t}), \psi(y, s)<y_{n}<\hat{x}_{n}\right\} .
$$

Since $d(\hat{X}, \hat{t}, \partial \Omega)=d(0, \hat{x}, \hat{t}, \partial \Omega)=d$, we may redefine $\tilde{\Omega}$ as

$$
\tilde{\Omega}=\left\{\left(y, y_{n}, s\right):(y, s) \in \operatorname{int} I_{c(n) d /(2 M)}(\hat{x}, \hat{t}), \psi(y, s)<y_{n}<\hat{x}_{n}+d / 2\right\} .
$$

By construction we have $\tilde{\Omega} \subset \Omega \cap Q_{2 C d}(\hat{X}, \hat{t})$. This proves that $\Omega$ satisfies a uniform interior big pieces of $\operatorname{Lip}(1,1 / 2)$ graphs with constants $(\epsilon, \hat{M}, C)$ where $\epsilon=\gamma, \hat{M}=2 M / c(n)$ and $C=C(n, M)$. Based on (2.1) we see that in the end all of these constants depend on $n, M$ and $\gamma_{0}$. This completes the proof of the second statement in Theorem 1.1.

2.1. Proof of Lemma 2.1. We let, for any

$$
(X, t) \in\left\{\left(x, x_{n}, t\right):(x, t) \in I^{0}, x_{n} \in[-M, \bar{M}]\right\},
$$

$L(X, t)$ be the open line segment in the $x_{n}$ direction which connects $(X, t)$ to $(x, \bar{M}, t)$. If $(X, t) \in \Sigma$, then the length of $L(X, t)$ is at least 1 as $d\left(I^{\bar{M}}, \Sigma\right) \geq 1$. Define $\tilde{\mathcal{G}}$ to be the closure of the set of all such points $(X, t) \in \Sigma$ which satisfy $L(X, t) \cap \Sigma=\emptyset$, and let

$$
\mathcal{G}:=\tilde{\mathcal{G}} \cap S \subset \Sigma .
$$

Consider $(x, t) \in I^{0}$ and define

$$
M(x, t)=\sup \left\{\frac{1}{|I|} \sigma\left(\pi^{-1}(I) \cap Q_{2 M}(0,0)\right): I \text { contains }(x, t)\right\} .
$$

Then, by weak estimates for the Hardy-Littlewood maximal function,

$$
\mathcal{B}:=\left\{(x, t) \in \mathbb{R}^{n}: M(x, t) \geq N\right\} \text { satisfies } \sigma(\mathcal{B}) \leq c / N,
$$

for some constant $c=c(n, M) \geq 1$. If $N$ is large then $\mathcal{B}$ contains those points $(x, t)$ which are such that there is a cube $I$, containing $(x, t)$, onto which a large set on $\Sigma$ is projected. Obviously there exists $N \geq 1$, depending only on $n, M$ and $\gamma$, such that

$$
\mathcal{H}^{n}(\mathcal{B}) \leq \gamma / 2 .
$$

We fix $N$ with respect to (2.12). In particular, both $\gamma$ and $N$ are from now on fixed constants depending only on $n, M$. 
Having fixed $\gamma$ and $N$, there will appear, in the construction to be outlined, four important constants: $\Lambda_{0}, \Lambda_{1}, \Lambda_{2}$, and $\Lambda_{3}$, with $1 \leq \Lambda_{i}<\infty$ for $i \in\{0,1,2,3\}$. In general all constants appearing will depend at most on $n, M, \Lambda_{0}, \Lambda_{1}, \Lambda_{2}$, and $\Lambda_{3}$. We will choose the degrees of freedom $\Lambda_{0}, \Lambda_{1}, \Lambda_{2}$, and $\Lambda_{3}$ to depend only on $n, M$, $\gamma$ and $N$, and hence to depend only on $n, M$. Furthermore, $\Lambda_{i}$ for $i \in\{0,1,2,3\}$, will be chosen to be of the form $2^{N_{i}}$ for some integer $N_{i} \geq 1$.

We let, for $\Lambda_{1}$ fixed and as above,

$$
A=\left[\log _{2}\left(2 M \Lambda_{1}\right)\right]+1 .
$$

Then $A$ is a power of 2 such that $A \geq 2 M \Lambda_{1}$. Based on $A$ we define, for $j \in\{0,1, \ldots\}$,

$\Sigma_{j}=\left\{(x, t) \in I^{0}:\right.$ there exist $(X, t) \in \mathcal{G}$ and $(Y, s) \in S$ such that

$$
\left.(X, t)=\left(x, x_{n}, t\right),(Y, s) \in(X, t)+\Gamma \text { and } A^{-j} \leq y_{n}-x_{n} \leq A^{-j+1}\right\} .
$$

If $\left(x, x_{n}, t\right) \in S$, there exists a maximal $\hat{x}_{n}$ such that $\left(x, \hat{x}_{n}, t\right) \in S$. This follows since $x_{n} \leq \bar{M}$ if $\left(x, x_{n}, t\right) \in S, I^{\bar{M}} \subset \mathcal{U}$ and $S$ is closed. Thus $\left(x, \hat{x}_{n}, t\right) \in \mathcal{G}$, which shows that $\pi(\mathcal{G})=\pi(S)$. When $(x, t) \in \pi(S) \backslash W$ we have $((X, t)+\Gamma) \cap S \neq \emptyset$ whenever $(X, t)=\left(x, x_{n}, t\right) \in S$. We conclude that for $(X, t)=\left(x, \hat{x}_{n}, t\right) \in \mathcal{G}$, $((X, t)+\Gamma) \cap S \neq \emptyset$, so there exists $(Y, s) \in S$ such that $(Y, s) \in(X, t)+\Gamma$. By our restriction on $A$ we have

$$
\pi(S) \backslash W \subset \cup_{j} \Sigma_{j}
$$

Furthermore, as by construction $\mathcal{H}^{n}(\mathcal{B}) \leq \gamma / 2$, the proof of Lemma 2.1 is reduced to proving that

$$
\mathcal{H}^{n}\left(\cup_{j} \Sigma_{j} \cap\left(\mathbb{R}^{n} \backslash \mathcal{B}\right)\right) \leq \gamma / 2 .
$$

To continue the proof we will need the following lemma, Lemma 2.2, the proof of which is postponed until subsection 2.2.

Lemma 2.2. Let $\varepsilon>0$ be given. Let $\Lambda_{1}$ be as above and define $A$ as in (2.13). Then there exist $\Lambda_{2}$, and $\Lambda_{3}$ as above and as in the statement of Lemma 2.5, and an integer $N_{0}=N_{0}\left(\epsilon, \Lambda_{2}\right) \geq 1$, such that if we let $\Lambda_{0}=\Lambda_{2}^{\tilde{N}_{0}}$, for some $\tilde{N}_{0} \geq N_{0}$, and if we restrict $h$ to satisfy $h \geq 2 A \Lambda_{0} \Lambda_{1} \Lambda_{3}$, then the following is true. Let $j \geq 0$ and $I \subset I^{0}$ be a dyadic cube of size $l(I)=A^{-j}$. Then the number of dyadic cubes $J$ of size $l(J)=\Lambda_{0}^{-1} A^{-j}$ that are contained in $I$ and satisfy $J \cap\left(\Sigma_{j} \cap\left(\mathbb{R}^{n} \backslash \mathcal{B}\right)\right) \neq \emptyset$, is less than $\varepsilon \Lambda_{0}^{n+1}$.

Let in the following $\epsilon>0$ be a degree of freedom to be fixed in (2.23) below. To proceed with the proof of (2.14), given $j \geq 0$ we dyadically subdivide $I^{0}$ into (disjoint) dyadic cubes $\left\{J_{j, l}\right\}$ of size $l\left(J_{j, l}\right)=A^{-j}$. Note that there are $A^{(n+1) j}$ such cubes. We then apply Lemma 2.2 to each cube $J_{j, l}$ and, as a result, for $\Lambda_{0}$ as in Lemma 2.2 we can produce a set of dyadic cube $\left\{J_{j, l, k}\right\}$, each of size $l\left(J_{j, l, k}\right)=\Lambda_{0}^{-1} A^{-j}$, such $J_{j, l, k} \subseteq J_{j, l}$ and such that $J_{j, l, k} \cap\left(\Sigma_{j} \cap\left(\mathbb{R}^{n} \backslash \mathcal{B}\right)\right) \neq \emptyset$. By Lemma 2.2 we have, for each $J_{j, l}$, that the number of such cubes is at most $\varepsilon \Lambda_{0}^{n+1}$. Using this we see that

$$
\begin{aligned}
\mathcal{H}^{n}\left(\cup_{j} \Sigma_{j} \cap\left(\mathbb{R}^{n} \backslash \mathcal{B}\right)\right) & \leq \sum_{j} \sum_{l} \mathcal{H}^{n}\left(J_{j, l} \cap\left(\Sigma_{j} \cap\left(\mathbb{R}^{n} \backslash \mathcal{B}\right)\right)\right) \\
& \leq \sum_{j} \sum_{l} \sum_{k=1}^{k_{l}} \mathcal{H}^{n}\left(J_{j, l, k}\right) .
\end{aligned}
$$

Hence, to prove (2.14) it suffices to prove that

$$
\sum_{j} \sum_{l} \sum_{k=1}^{k_{l}} \mathcal{H}^{n}\left(J_{j, l, k}\right) \leq \gamma / 2 .
$$


In the following we will, to simplify the notation, simply denote the set of all cubes $\left\{J_{j, l, k}\right\}$ by $\left\{I_{j, k}\right\}$. To each $I_{j, k}$ we will associate a surface $S\left(I_{j, k}\right)$, see Definition 10 below, and we intend to estimate the measure of $I_{j, k}$ in terms of the measure of $S\left(I_{j, k}\right)$. The surfaces $\left\{S\left(I_{j, k}\right)\right\}$ will not be uniquely defined, but if $j \neq j^{\prime}$, then we will have $S\left(I_{j, k}\right) \cap S\left(I_{j^{\prime}, k^{\prime}}\right)=\emptyset$ for all $k$ and $k^{\prime}$, see Lemma 2.3 below. For the sake of brevity we will in the following denote points $(X, t)=\left(x, x_{n}, t\right)$ by boldface letters, i.e., $\mathbf{X}:=(X, t)=\left(x, x_{n}, t\right)$. To proceed with the construction, consider $I=I_{j, k}$ and choose any $\mathbf{X} \in \mathcal{G}$ and $\mathbf{Y} \in S$ such that $\pi(\mathbf{X}) \in I, \mathbf{Y} \in \mathbf{X}+\Gamma$ and $A^{-j} \leq y_{n}-x_{n}<A^{-j+1}$. Applying the synchronized two-cube condition we see that there exists a cube $Q \subset \mathbb{R}^{n+1}$ of size $R_{j}=M^{-1} \Lambda_{1}^{-1} A^{-j}$ and with center $\mathbf{U}$, such that $\|\mathbf{U}-\mathbf{Y}\| \leq \Lambda_{1}^{-1} A^{-j}$ and such that $Q$ belongs to a component of $\mathbb{R}^{n+1} \backslash \Sigma$ different from $\mathcal{U}$. We recall that $\mathcal{U}$ is the component that contains $I^{\bar{M}}$. Furthermore, the $t$-coordinate of $\mathbf{U}$ and $\mathbf{Y}$, coincide. Given $I$ we let $J=J(I)=I_{\Lambda_{1}^{-1} R_{j}}(\pi(\mathbf{U}))$ and $\mathcal{S}=\left(\Sigma \cap Q_{2 M}(0,0)\right) \cup\left(I^{0} \times\left\{x_{n}=-A\right\}\right)$.

Definition 10. Given $I=I_{j, k}$ we define $S(I)$ to be the set of all $\mathbf{V} \in \mathcal{S}$ such that $\pi(\mathbf{V}) \in J=J(I)=I_{\Lambda_{1}^{-1} R_{j}}(\pi(\mathbf{U})), v_{n}<u_{n}-R_{j}$ and such that the line segment joining $\mathbf{V}$ to $\pi(\mathbf{V})+\left(0, u_{n}-R_{j}, 0\right)$ does not meet $\Sigma$.

Note that $\pi(S(I))=J$ as $\pi(\mathcal{S}) \supset J$. Moreover, it is easy to check that $J \subset 2 I$. To complete the proof we will need the following lemma, Lemma 2.3, the proof of which is also postponed until subsection 2.2.

Lemma 2.3. Let $\varepsilon>0$ be given. Let $\Lambda_{2}, \Lambda_{3}, N_{0}$, be as in the statement of Lemma 2.2. Then there exists an integer $\tilde{N}_{0} \geq N_{0}$, depending only on $n, M, \Lambda_{2}, \Lambda_{3}$, such that if we let $\Lambda_{0}=\Lambda_{2}^{\tilde{N}_{0}}, \Lambda_{1}=2^{\tilde{N}_{0}}$, define $A$ as in (2.13), and if we restrict $h$ to satisfy $h \geq 2 A \Lambda_{0} \Lambda_{1} \Lambda_{3}$, then

$$
S\left(I_{j, k}\right) \cap S\left(I_{j^{\prime}, k^{\prime}}\right)=\emptyset \text { for all } k \text { and } k^{\prime} \text { whenever } j \neq j^{\prime} .
$$

We can now use Lemma 2.2 and Lemma 2.3 to complete the proof of (2.16) and hence the proof of Lemma 2.1. Let $K_{j}$ be the number of cubes $J_{j, l}$ that contain at least one of the $J_{j, l, k}$. Then, given $\epsilon, \tilde{N}_{0} \geq N_{0}$ and $h$ as stated in Lemma 2.3, we see, using Lemma 2.2, that

(2.17) $\sum_{l} \sum_{k=1}^{k_{l}} \mathcal{H}^{n}\left(J_{j, l, k}\right) \leq K_{j} \varepsilon \Lambda_{0}^{n+1}\left(2 \Lambda_{0}^{-1} A^{-j}\right)^{n+1}=K_{j} \varepsilon 2^{n+1} A^{-j(n+1)}$.

Fix $l$ and assume that $J_{j, l} \supset J_{j, l, k}$ for some $k$. Then for all $k \in\left\{1, \ldots, k_{l}\right\}$ one has $\pi\left(S\left(J_{j, l, k}\right)\right) \subset 2 J_{j, l}$. By construction

$$
\mathcal{H}^{n}\left(\pi\left(\cup_{k=1}^{k_{l}} S\left(J_{j, l, k}\right)\right) \cap 2 J_{j, l}\right) \geq\left(2 \Lambda_{1}^{-1} R_{j}\right)^{n+1} .
$$

Hence, summing over $l$ we see that

$$
\begin{aligned}
\sum_{l} \mathcal{H}^{n}\left(\pi\left(\cup_{k=1}^{k_{l}} S\left(J_{j, l, k}\right)\right) \cap 2 J_{j, l}\right) & \geq K_{j}\left(2 \Lambda_{1}^{-1} R_{j}\right)^{n+1} \\
& =K_{j}\left(M^{-1} \Lambda_{1}^{-2}\right)^{n+1} 2^{n+1} A^{-j(n+1)} .
\end{aligned}
$$

Combining (2.17) and (2.19) we see that

$$
\sum_{l} \sum_{k=1}^{k_{l}} \mathcal{H}^{n}\left(J_{j, l, k}\right) \leq c \epsilon\left(M \Lambda_{1}^{2}\right)^{n+1} \sum_{l} \mathcal{H}^{n}\left(\pi\left(\cup_{k=1}^{k_{l}} S\left(J_{j, l, k}\right)\right)\right)
$$

for all $j \geq 0$, where the constant $c=c(n)$ comes from the possible overlap between the sets $2 J_{j, l}$ in (2.19). Next, using Lemma 2.3 we know that the $S\left(J_{j, l, k}\right) \cap$ $S\left(J_{j^{\prime}, l, k}\right)=\emptyset$ whenever $j \neq j^{\prime}$. Furthermore, by definition,

$$
S\left(J_{j, l, k}\right) \subset \mathcal{S}=\left(\Sigma \cap Q_{2 M}(0,0)\right) \cup\left(I^{0} \times\left\{x_{n}=-A\right\}\right) .
$$


This implies that

$$
\begin{aligned}
\sum_{j} \sum_{l} \mathcal{H}^{n}\left(\pi\left(\cup_{k=1}^{k_{l}} S\left(J_{j, l, k}\right)\right)\right) & \leq \mathcal{H}^{n}\left(\pi(\Sigma) \cap I^{0}\right)+\mathcal{H}^{n}\left(\pi\left(I^{0} \times\left\{x_{n}=-A\right\}\right)\right) \\
& =2 \mathcal{H}^{n}\left(I^{0}\right) .
\end{aligned}
$$

Together (2.20) and (2.21) imply the bound

$$
\sum_{l} \sum_{k=1}^{k_{l}} \mathcal{H}^{n}\left(J_{j, l, k}\right) \leq C \epsilon\left(M \Lambda_{1}^{2}\right)^{n+1},
$$

where $C=C(n), 1 \leq C<\infty$. Let now $\epsilon$ be defined through the relation

$$
C \epsilon\left(M \Lambda_{1}^{2}\right)^{n+1}=\gamma / 2 \text {. }
$$

Then $\varepsilon=\varepsilon\left(n, M, \Lambda_{1}, \gamma\right)=\varepsilon(n, M, \gamma)=\varepsilon(n, M)$ and we see that Lemma 2.1 holds with $h=2 A \Lambda_{0} \Lambda_{1} \Lambda_{3}$ and, by construction, $h=h(n, M)$. In particular, the proof of Lemma 2.1 is now, modulo Lemma 2.2 and Lemma 2.3, complete.

\subsection{Proof of Lemma 2.2 and Lemma 2.3.}

Lemma 2.4. Let $I \subset I^{0}$ be a cube of size $l(I)$. Suppose there exist two line segments $L$ and $L^{\prime}$ in different components of $\mathbb{R}^{n+1} \backslash \Sigma$ such that $\pi(L) \in I, \pi\left(L^{\prime}\right) \in I$ and such that the length of the line segment $\pi^{\perp}(L) \cap \pi^{\perp}\left(L^{\prime}\right) \cap(-3 M / 2,3 M / 2)$ is greater than $4^{n+2} M N l(I)$. Then $2 I \subset \mathcal{B}$.

Proof. Any line segment orthogonal to $x_{n}$ that connects $L$ and $L^{\prime}$ intersects $\Sigma$, and by construction there exist $4^{n+1} M N$ such line segments with mutual distance greater than $2 l(I)$. Thus there exist $4^{n+1} M N$ points $\left(X^{i}, t^{i}\right) \in \Sigma$ such that $\pi\left(X^{i}, t^{i}\right) \in I$ and $\left\|\pi^{\perp}\left(X^{i}, t^{i}\right)-\pi^{\perp}\left(X^{j}, t^{j}\right)\right\| \geq 2 l(I)$ for $i \neq j$. Hence the cubes $Q_{l(I)}\left(X^{i}, t^{i}\right)$ are disjoint and satisfy $\pi\left(Q_{l(I)}\left(X^{i}, t^{i}\right)\right) \subset 2 I$. This gives the estimate

$$
\begin{aligned}
\sigma\left(\pi^{-1}(2 I) \cap Q_{2 M}(0,0)\right) & \geq \sigma\left(\bigcup_{i} Q_{l(I)}\left(X^{i}, t^{i}\right)\right) \\
& \geq 4^{n+1} M N M^{-1} l(I)^{n+1} \geq N \mathcal{H}^{n}(2 I) .
\end{aligned}
$$

This completes the proof of the lemma.

Lemma 2.5. Let $h \geq 1$ be given. There exist constants $\Lambda_{2}=2^{N_{2}}$ and $\Lambda_{3}=2^{N_{3}}$, where $N_{2}$ and $N_{3}$ are positive integers, depending only on $n, M$ and $N$, such that the following holds. If $I \subset I^{0}$ is a dyadic cube of size $l(I), \Lambda_{3} h^{-1} A^{-j+1} \leq l(I) \leq A^{-j}$, then there is a dyadic cube $J$ of size $\Lambda_{2}^{-1} l(I)$ such that $J \subset \mathcal{B} \cup\left(\mathbb{R}^{n} \backslash \Sigma_{j}\right)$.

Proof. We first note that it suffices to prove the existence of cube $J$, as in the statement of the lemma, but which is not dyadic, as $J$ then contains a dyadic cube of size $2^{-1} \Lambda_{2}^{-1} l(I)$ and we may replace $\Lambda_{2}$ by $2 \Lambda_{2}$. Furthermore, we in the following just establish the existence of $\Lambda_{2}$ and $\Lambda_{3}$. That these constants can be chosen on the form $\Lambda_{2}=2^{N_{2}}$ and $\Lambda_{3}=2^{N_{3}}$ is then obvious. Given $I$ we let $K \geq 4$ be a degree of freedom, which we fix in (2.25) and which only depends on $n$ and $M$, and in the following we let $J_{k}$, for $k \geq 1$, denote the cube which has the same center as $I$ but which has size $2^{-k} l(I)$. By construction

$$
I \supset J_{1} \supset J_{2} \supset \cdots \supset J_{k} \supset \cdots .
$$

Assume that $J_{k} \cap \pi(\mathcal{G})=\emptyset$ for some $k \in\{1, \ldots, K+1\}$. Then $J_{k} \subset\left(\mathbb{R}^{n} \backslash \Sigma_{j}\right)$ and we can choose $J=J_{K+1}, \Lambda_{2}=2^{(K+1)}, \Lambda_{3}=1$, to complete the proof of the lemma. Hence we in the following assume that $\mathrm{f} J_{k} \cap \pi(\mathcal{G}) \neq \emptyset$ for all $1 \leq k \leq K+1$. In this case we introduce

$$
\lambda_{k}=\sup \left\{x_{n}: \mathbf{X} \in \mathcal{G} \text { and } \pi(\mathbf{X}) \in J_{k}\right\} .
$$


Obviously $\left\{\lambda_{k}\right\}$ is a non-increasing sequence. Assume first that $\lambda_{k}>\lambda_{k+1}+A^{-j} / 10$ for all $k \in\{2, \ldots, K\}$. Then there exist $K$ points $\mathbf{X}^{k} \in \mathcal{G}$ such that $\pi\left(\mathbf{X}^{k}\right) \in J_{k}$ and

$$
\left\|\mathbf{X}^{k}-\mathbf{X}^{k^{\prime}}\right\| \geq\left|x_{n}^{k}-x_{n}^{k^{\prime}}\right|>A^{-j} / 10, \quad k \neq k^{\prime} .
$$

Using that $\Sigma$ is parabolic ADR we see that

$$
\begin{aligned}
\sigma\left(\pi^{-1}(I)\right) & \geq \sum_{k=1}^{K} \sigma\left(Q_{A^{-j} / 20}\left(\mathbf{X}^{k}\right) \cap \Sigma\right) \\
& \geq K M^{-1} A^{-j(n+1)} 20^{-(n+1)} \geq c^{-1} K M^{-1} \mathcal{H}^{n}(I) .
\end{aligned}
$$

Hence, if we choose

$$
K=2 c M N,
$$

then $I \subset \mathcal{B}$ by the definition of the set $\mathcal{B}$. In this case we can again choose $J=J_{K+1}, \Lambda_{2}=2^{(K+1)}, \Lambda_{3}=1$, to complete the proof of the lemma. Next, assume that $\lambda_{k}<\lambda_{k+1}+A^{-j} / 10$ for some $k \in\{2, \ldots, K\}$ and choose $\mathbf{X} \in \mathcal{G}$ satisfying $\pi(\mathbf{X}) \in J_{k+1}, x_{n}=\lambda_{k+1}$. As $\mathbf{X} \in \Sigma$ we can use the synchronized two-cube condition to conclude that there is a cube $Q$ with size

$$
l(Q)=(10 M)^{-1} 2^{-k} l(I)
$$

and center $\mathbf{Y}$, having the same $t$-coordinate as $\mathbf{X}$, such that $\|\mathbf{X}-\mathbf{Y}\| \leq 2^{-k} l(I) / 10$ and such that $Q$ belongs to a component $\mathcal{V}$, different from $\mathcal{U}$, of $\mathbb{R}^{n+1} \backslash \Sigma$. In this case we let $J \subset I^{0}$ be the cube with center $\pi(\mathbf{Y})$ and size

$$
l(J)=\left(4^{n+1} M N\right)^{-1} l(Q) .
$$

If $J \cap \Sigma_{j}=\emptyset$, then the conclusion of the lemma holds with this choice of $J$, with $\Lambda_{2}$ defined through the relation

$$
\Lambda_{2}^{-1}=\left(4^{n+1} M N\right)^{-1}(10 M)^{-1} 2^{-K},
$$

and with $\Lambda_{3}=1$. If $J \cap \Sigma_{j} \neq \emptyset$, then there exist $\mathbf{U} \in \mathcal{G}, \mathbf{V} \in S$ such that $\pi(\mathbf{U}) \in J$, $\mathbf{V} \in \mathbf{U}+\Gamma$ and $A^{-j} \leq v_{n}-u_{n} \leq A^{-j+1}$. From the definition of $\Gamma$ we get

$$
\|\pi(\mathbf{U})-\pi(\mathbf{V})\| \leq h^{-1}\left(v_{n}-u_{n}\right) \leq h^{-1} A^{-j+1} \leq \Lambda_{3}^{-1} l(I),
$$

provided $l(I) \geq \Lambda_{3} h^{-1} A^{-j+1}$. Hence, if we choose

$$
\Lambda_{3}=100 M^{2} 4^{n+1} N 2^{K},
$$

then

$$
\|\pi(\mathbf{U})-\pi(\mathbf{V})\| \leq l(J) / 10 .
$$

We claim that $\pi(\mathbf{V}) \in J_{k}$. Indeed, recall that $\pi(\mathbf{X}) \in J_{k+1}$ and

$$
\|\pi(\mathbf{V})-\pi(\mathbf{X})\| \leq\|\pi(\mathbf{X})-\pi(\mathbf{Y})\|+\|\pi(\mathbf{Y})-\pi(\mathbf{U})\|+\|\pi(\mathbf{U})-\pi(\mathbf{V})\|
$$

where

$$
\begin{aligned}
&\|\pi(\mathbf{X})-\pi(\mathbf{Y})\| \leq\|\mathbf{X}-\mathbf{Y}\|=2^{-k} l(Q) / 10, \\
&\|\pi(\mathbf{Y})-\pi(\mathbf{U})\| \leq(n-1)^{1 / 2} l(J)+l(J) \leq n l(J),
\end{aligned}
$$

and

$$
\|\pi(\mathbf{U})-\pi(\mathbf{V})\| \leq l(J) / 10 .
$$

Hence, using this, (2.28) and the definitions of $l(Q)$ and $l(J)$ we see that

$$
\|\pi(\mathbf{V})-\pi(\mathbf{X})\|<\frac{1}{2} 2^{-(k+1)} r .
$$

Therefore $\pi(\mathbf{V}) \in J_{k}$ as $\pi(\mathbf{X}) \in J_{k+1}$. Thus

$$
v_{n} \leq \lambda_{k} \leq \lambda_{k+1}+A^{-j} / 10=x_{n}+A^{-j} / 10 .
$$


Using this, and that $u_{n} \leq v_{n}-A^{-j}$ and $\|\mathbf{Y}-\mathbf{X}\|<A^{-j} / 10$, we conclude that

$$
u_{n} \leq x_{n}-9 A^{-j} / 10 \leq y_{n}-8 A^{-j} / 10 \text {. }
$$

Furthermore, as $\mathbf{U} \in \mathcal{G}$ there exists a point $\mathbf{U}^{\prime}$, arbitrarily close to $\mathbf{U}$, such that the vertical line segment $L^{\prime}$ connecting $\mathbf{U}^{\prime}$ to $I^{\bar{M}}$ belongs to $\mathcal{U}$. Choose $\mathbf{U}^{\prime}$ such that $\left\|\mathbf{U}-\mathbf{U}^{\prime}\right\|<l(J) / 10$. Then $\pi\left(\mathbf{U}^{\prime}\right) \in 2 J$. Let $L$ be the line segment of length $2 l(Q)$ which is the intersection between $Q$ and the line parallel to $L^{\prime}$ through $\mathbf{Y}$. By construction $L$ and $L^{\prime}$ belong to different components of $\mathbb{R}^{n+1} \backslash \Sigma$ and the length of $L^{\prime}$ is greater than $1 / 2$ since $d\left(I^{\bar{M}}, \Sigma\right) \geq 1$. Moreover,

$$
u_{n}^{\prime} \leq y_{n}-8 A^{-j} / 10+l(J) / 10 \leq y_{n}-7 A^{-j} / 10<y_{n}-l(Q),
$$

which shows that the length of $\pi^{\perp}(L) \cap \pi^{\perp}\left(L^{\prime}\right)$ is $2 l(Q)$. As $l(Q) \geq 4^{n+1} M N l(J)>$ $2^{n+1} M N(2 l(J))$ we conclude, using Lemma 2.4 , that $4 J \subset \mathcal{B}$. Hence in this case we can complete the proof by choosing this $J, \Lambda_{2}$ as in (2.26) and $\Lambda_{3}$ as in (2.27). In particular, the conclusion holds in all cases with

$$
\Lambda_{2}=\left(4^{n+1} M N\right)(10 M) 2^{K}, \Lambda_{3}=100 M^{2} 4^{n+1} N 2^{K} .
$$

This completes the proof of the lemma.

Proof of Lemma 2.2. Let $\Lambda_{2}$ be the constant from Lemma 2.5. Lemma 2.5 guarantees, if $\Lambda_{3} h^{-1} A^{-j+1} \leq l(I) \leq A^{-j}$, that at least one of the $\Lambda_{2}^{(n+1)}$ dyadic cubes of size $\Lambda_{2}^{-1} A^{-j}$ covering $I$ does not intersect $\Sigma_{j} \cap\left(\mathbb{R}^{n} \backslash \mathcal{B}\right)$. If we apply Lemma 2.5 again to the remaining cubes it follows, if $\Lambda_{3} h^{-1} A^{-j+1} \leq \Lambda_{2}^{-1} l(I) \leq A^{-j}$, that the number of dyadic cubes of size $\Lambda_{2}^{-2} A^{-j}$ that intersect $\Sigma_{j} \cap\left(\mathbb{R}^{n} \backslash \mathcal{B}\right)$ is bounded by $\left(\Lambda_{2}^{n+1}-1\right)^{2}$. Repeating this procedure $\tilde{N}_{0}$ times, we see, if $\Lambda_{3} h^{-1} A^{-j+1} \leq$ $\Lambda_{2}^{-\tilde{N}_{0}+1} l(I) \leq A^{-j}$, that the number of dyadic cubes of size $\Lambda_{2}^{-\tilde{N}_{0}} A^{-j}$ that intersect $\Sigma_{j} \cap\left(\mathbb{R}^{n} \backslash \mathcal{B}\right)$ is bounded by

$$
\left(\Lambda_{2}^{n+1}-1\right)^{\tilde{N}_{0}}=\Lambda_{2}^{\tilde{N}_{0}(n+1)}\left(1-\Lambda_{2}^{-n-1}\right)^{\tilde{N}_{0}} .
$$

Let $N_{0}=N_{0}\left(\varepsilon, \Lambda_{2}\right)$ be the smallest positive integer such that

$$
\left(\Lambda_{2}^{n+1}-1\right)^{N_{0}} \leq \varepsilon \Lambda_{0}^{n+1} .
$$

Consider $\tilde{N}_{0} \geq N_{0}$ and let $\Lambda_{0}=\Lambda_{2}^{\tilde{N}_{0}}$. Then

$$
\left(\Lambda_{2}^{n+1}-1\right)^{\tilde{N}_{0}} \leq \epsilon \Lambda_{0}^{(n+1)} .
$$

Furthermore, we see that our choice of $h$ implies that

$$
\Lambda_{3} h^{-1} A \leq \Lambda_{2}^{-\tilde{N}_{0}} / 2 \leq \Lambda_{2}^{-\tilde{N}_{0}+1},
$$

and hence, as $l(I)=A^{-j}$, we can ensure that $\Lambda_{3} h^{-1} A^{-j+1} \leq \Lambda_{2}^{-\tilde{N}_{0}+1} l(I) \leq A^{-j}$. This completes the proof of the lemma.

Proof of Lemma 2.3. To prove the lemma we argue by contradiction. Indeed, assume that there exist $I=I_{j, k}, I^{\prime}=I_{j^{\prime}, k^{\prime}}, j>j^{\prime}$, such that $S(I) \cap S\left(I^{\prime}\right) \neq \emptyset$. Let $\mathbf{V} \in S(I) \cap S\left(I^{\prime}\right)$. Let $\mathbf{X}, \mathbf{Y}, \mathbf{U}$ and $Q$ be associated to the construction of $S(I)$ and let $\mathbf{X}^{\prime}, \mathbf{Y}^{\prime}, \mathbf{U}^{\prime}$ and $Q^{\prime}$ be associated to the construction of $S\left(I^{\prime}\right)$, see Definition 10 and the notation introduced above that definition. We have

$$
\begin{aligned}
\|\pi(\mathbf{X})-\pi(\mathbf{V})\| & \leq\|\pi(\mathbf{X})-\pi(\mathbf{Y})\|+\|\pi(\mathbf{Y})-\pi(\mathbf{V})\| \\
& \leq h^{-1} A^{-j+1}+\|\pi(\mathbf{Y})-\pi(\mathbf{V})\|,
\end{aligned}
$$

and

$$
\|\pi(\mathbf{Y})-\pi(\mathbf{V})\| \leq\|\mathbf{Y}-\mathbf{U}\|+\|\pi(\mathbf{U})-\pi(\mathbf{V})\| \leq \Lambda_{1}^{-1} A^{-j}+n \Lambda_{1}^{-1} R_{j} .
$$

Thus

$$
\|\pi(\mathbf{X})-\pi(\mathbf{V})\| \leq h^{-1} A^{-j+1}+\Lambda_{1}^{-1} A^{-j}+n M^{-1} \Lambda_{1}^{-2} A^{-j}
$$




$$
\leq\left(h^{-1} A+\Lambda_{1}^{-1}+n M^{-1} \Lambda_{1}^{-2}\right) A^{-j} \leq 2 \Lambda_{1}^{-1} A^{-j}
$$

by our choices for $M$ and $h$. The distance between $\pi(\mathbf{X})$ and $\pi\left(\mathbf{U}^{\prime}\right)$ can be estimated as

$$
\begin{aligned}
\left\|\pi(\mathbf{X})-\pi\left(\mathbf{U}^{\prime}\right)\right\| & \leq\|\pi(\mathbf{X})-\pi(\mathbf{V})\|+\left\|\pi(\mathbf{V})-\pi\left(\mathbf{U}^{\prime}\right)\right\| \\
& \leq 2 \Lambda_{1}^{-1} A^{-j}+n \Lambda_{1}^{-1} R_{j^{\prime}} .
\end{aligned}
$$

By construction, $A>2 M \Lambda_{1}$, which implies that $2 \Lambda_{1}^{-1} A^{-j} \leq 2 \Lambda_{1}^{-1} A^{-1} A^{-j^{\prime}} \leq$ $\Lambda_{1}^{-1} R_{j^{\prime}}$ and that

$$
\left\|\pi(\mathbf{X})-\pi\left(\mathbf{U}^{\prime}\right)\right\| \leq(n+1) \Lambda_{1}^{-1} R_{j^{\prime}}
$$

Hence we can conclude that $\pi(\mathbf{X}) \in(n+1) I_{\Lambda_{1}^{-1} R_{j^{\prime}}}\left(\pi\left(\mathbf{U}^{\prime}\right)\right)$. As $\mathbf{X} \in \mathcal{G}$, there is a point $\mathbf{W}$ such that $\|\mathbf{W}-\mathbf{X}\| \leq \Lambda_{1}^{-1} R_{j}$ and such that the vertical line $L$ connecting $\mathbf{W}$ to $I^{\bar{M}}$ belongs to $\mathcal{U}$. Note that $\left\|\pi(\mathbf{W})-\pi\left(\mathbf{U}^{\prime}\right)\right\| \leq(n+2) \Lambda_{1}^{-1} R_{j^{\prime}}$. We will now consider the two possibilities $x_{n}<u_{n}^{\prime}-R_{j^{\prime}}$ and $x_{n} \geq u_{n}^{\prime}-R_{j^{\prime}}$ separately, and will derive a contradiction to the assumption that there exists a point $\mathbf{V} \in S(I) \cap S\left(I^{\prime}\right)$.

Assume first that $x_{n}<u_{n}^{\prime}-R_{j^{\prime}}$, let $L^{\prime}$ be the vertical line segment in $Q^{\prime}$ through $\mathbf{U}^{\prime}$ of length $2 R_{j^{\prime}}$. Then the projections of $L$ and $L^{\prime}$ belong to $(n+$ 2) $I_{\Lambda_{1}^{-1} R_{j^{\prime}}}\left(\pi\left(\mathbf{U}^{\prime}\right)\right)$. Furthermore, as $\|\mathbf{W}-\mathbf{X}\|<R_{j^{\prime}}$ it follows that the length of the line segment $\pi^{\perp}\left(L^{\prime}\right) \cap \pi^{\perp}(L)$ is greater than $R_{j^{\prime}}$. We now want to apply Lemma 2.4. Indeed, if $R_{j^{\prime}}>4^{n+2} M N(n+2) \Lambda_{1}^{-1} R_{j^{\prime}}$ then Lemma 2.4 implies that $(n+2) I_{\Lambda_{1}^{-1} R_{j^{\prime}}}\left(\pi\left(\mathbf{U}^{\prime}\right)\right) \subset \mathcal{B}$ if we choose $\tilde{N}_{0}$, and hence $\Lambda_{1}$, is large enough. Furthermore, $\pi(\mathbf{X}) \in I \cap(n+1) I_{\Lambda_{1}^{-1} R_{j^{\prime}}}\left(\pi\left(\mathbf{U}^{\prime}\right)\right)$, and as the size of $I$ is $\Lambda_{0}^{-1} A^{-j}$, we have $\left.I \subset(n+2) I_{\Lambda_{1}^{-1} R_{j^{\prime}}}\left(\pi\left(\mathbf{U}^{\prime}\right)\right)\right) \subset \mathcal{B}$ if $\tilde{N}_{0}$, and hence $\Lambda_{0}$, is large enough. This contradicts the assumption that $I$ meets $\Sigma_{j} \cap\left(\mathbb{R}^{n} \backslash \mathcal{B}\right)$.

Assume next that $x_{n} \geq u_{n}^{\prime}-R_{j^{\prime}}$. Let

$$
\mathbf{Z}=\pi(\mathbf{V})+\left(0, u_{n}-\Lambda_{1}^{-1} R_{j}, 0\right)
$$

and let $L^{\prime \prime}$ be the line segment joining $\mathbf{V}$ and $\mathbf{Z}$. Then $L^{\prime \prime}$ does not meet $\Sigma$ since $\mathbf{V} \in S(I)$, and $L^{\prime \prime}$ belongs to a component of $\mathbb{R}^{n+1} \backslash \Sigma$ other than $\mathcal{U}$ since it intersects $Q$. We have

$$
\begin{aligned}
z_{n} & =u_{n}-\Lambda_{1}^{-1} R_{j} \geq y_{n}-\Lambda_{1}^{-1} A^{-j}-\Lambda_{1}^{-1} R_{j} \geq y_{n}-2 \Lambda_{1}^{-1} A^{-j} \\
& \geq x_{n}+A^{-j}-2 \Lambda_{1}^{-1} A^{-j} \geq x_{n}+A^{-j} / 2 \geq w_{n}+A^{-j} / 4 .
\end{aligned}
$$

Since $\mathbf{V} \in S\left(I^{\prime}\right)$, we also have

$$
v_{n} \leq u_{n}^{\prime}-R_{j^{\prime}} \leq x_{n}
$$

By (2.31) and (2.32), we have $z_{n}-v_{n} \geq A^{-j} / 2$. Thus the length of $L^{\prime \prime}$ is at least $A^{-j} / 2$, and the length of $\pi^{\perp}\left(L^{\prime \prime}\right) \cap \pi^{\perp}(L)$ is at least $A^{-j} / 4$, again by $(2.31)$. Since $\|\pi(\mathbf{V})-\pi(\mathbf{W})\| \leq 3 \Lambda_{1}^{-1} A^{-j}$, we conclude, using Lemma 2.4, that the cube $I^{\mathbf{W}}$ of size $3 \Lambda_{1}^{-1} A^{-j}$ and center $\pi(\mathbf{W})$ belongs to $\mathcal{B}$ if $\tilde{N}_{0}$, and hence $\Lambda_{1}$, is large enough. If $\tilde{N}_{0}$, and hence $\Lambda_{0}$, is large enough we again arrive at the contradiction $I \subset I^{\mathbf{W}} \subset \mathcal{B}$.

\section{The proof of Theorem 1.2}

We here prove Theorem 1.2. To prove the first statement in Theorem 1.2 we note that we can conclude, by the first statement in Theorem 1.1, that there exists, given $(X, t) \in \Sigma, R>0$, and after a possible rotation in the space variables, a Lip $(1,1 / 2)$ function $\hat{\psi}$ with constant $\hat{M}=\hat{M}(n, M)$, and $\epsilon=\epsilon(n, M)$, such that if we let $\pi$ denote the orthogonal projection onto the plane $\left\{\left(x, x_{n}, t\right) \in \mathbb{R}^{n-1} \times \mathbb{R} \times \mathbb{R}: x_{n}=0\right\}$, then

$$
\mathcal{H}^{n}(\pi(F)) \geq \epsilon R^{n+1}, F:=\Sigma_{\hat{\psi}} \cap \Delta(X, t, R),
$$


where

$$
\Sigma_{\hat{\psi}}=\left\{\left(x, x_{n}, t\right) \in \mathbb{R}^{n-1} \times \mathbb{R} \times \mathbb{R}: x_{n}=\hat{\psi}(x, t)\right\} .
$$

To prove Theorem 1.2 we need to invoke the Carleson measure condition used in the very definition of parabolic uniform rectifiability. Let

$$
f(Z, \tau)=\int_{0}^{100 R} \gamma(Z, \tau, r) r^{-1} d r,(Z, \tau) \in \Delta(X, t, R) .
$$

Then, using (1.9) we see that

$$
\int_{\Delta(X, t, 100 R)} f(Z, \tau) d \sigma(Z, \tau) \leq\|\nu\|(100 R)^{n+1}
$$

where $\|\nu\|$ is the Carleson norm of $\nu$ on $\Delta(X, t, 800 R) \times(0,800 R)$. Using this and weak estimates we see that if $A=1000 \epsilon^{-1}$, then

$$
\begin{aligned}
\sigma\left(\left\{(Z, \tau) \in \Delta(X, t, 100 R): f(Z, \tau) \geq A^{n+1}\|\nu\|\right\}\right) & \leq(100 R / A)^{n+1} \\
& \leq(\epsilon R / 10)^{n+1}
\end{aligned}
$$

Using this inequality, (3.1) and the fact that Hausdorff measure does not increase under a projection, we deduce the existence of a closed set $F_{1}=F_{1}(A)$ with $F_{1} \subset F$, such that

$$
f(Z, \tau) \leq A^{n+1}\|\nu\|,(Z, \tau) \in F_{1}
$$

and

$$
\mathcal{H}^{n}\left(\pi\left(F_{1}\right)\right) \geq \frac{\epsilon}{2} R^{n+1}
$$

We will now construct the approximating graph by extending $\hat{\psi}$ off $\pi\left(F_{1}\right)$. To do this we again identify $\mathbb{R}^{n}$ with $\mathbb{R}^{n} \times\{0\}$, and put

$$
I_{r}(z, \tau)=\left\{(y, s) \in \mathbb{R}^{n}:\left|y_{i}-z_{i}\right|<r, i=1, \ldots, n-1,|s-\tau|<r^{2}\right\},
$$

whenever $(z, \tau) \in \mathbb{R}^{n}, r>0$. Let $\left\{\bar{I}_{i}=\overline{I_{r_{i}}\left(\hat{x}_{i}, \hat{t}_{i}\right)}\right\}$ be a Whitney decomposition of $\mathbb{R}^{n} \backslash \pi\left(F_{1}\right)$ into cubes, such that $I_{i} \cap I_{j}=\emptyset, i \neq j$, and

$$
10^{-10 n} d\left(I_{i}, \pi\left(F_{1}\right)\right) \leq r_{i} \leq 10^{-8 n} d\left(I_{i}, \pi\left(F_{1}\right)\right) .
$$

Let $\left\{v_{i}\right\}$ be a partition of unity adapted to $\left\{I_{i}\right\}$, i.e.,

(a) $\quad \sum v_{i} \equiv 1$ on $\mathbb{R}^{n} \backslash \pi\left(F_{1}\right)$,

(b) $\quad v_{i} \equiv 1$ on $I_{i}$ and $v_{i} \equiv 0$ in $\mathbb{R}^{n} \backslash \overline{I_{2 r_{i}}\left(\hat{x}_{i}, \hat{t}_{i}\right)}$ for all $i$,

(c) $\quad v_{i}$ is infinitely differentiable on $\mathbb{R}^{n}$ with

$$
r_{i}^{-l}\left|\frac{\partial^{l}}{\partial x^{l}} v_{i}\right|+r_{i}^{-2 l}\left|\frac{\partial^{l}}{\partial t^{l}} v_{i}\right| \leq c(l, n) \text { for } l=1,2, \ldots
$$

In $(c), \frac{\partial^{l}}{\partial x^{l}}$ denotes an arbitrary partial derivative with respect to the space variable $x$ and of order $l$. Next for each $i$ we choose $\left(x_{i}^{\prime}, t_{i}^{\prime}\right) \in \pi\left(F_{1}\right)$ with

$$
\rho_{i}=d\left(\left\{\left(x_{i}^{\prime}, t_{i}^{\prime}\right)\right\}, I_{i}\right)=d\left(\pi\left(F_{1}\right), I_{i}\right),
$$

and we set $\Lambda=\left\{i: \bar{I}_{i} \cap \overline{I_{2 R}(x, t)} \neq \emptyset\right\}$, where $(x, t)$ is the projection of $(X, t)$ onto $\mathbb{R}^{n}$. We now let

$$
\psi(y, s)=\left\{\begin{array}{l}
\hat{\psi}(y, s),(y, s) \in \pi\left(F_{1}\right) \\
\sum_{i \in \Lambda}\left(\hat{\psi}\left(x_{i}^{\prime}, t_{i}^{\prime}\right)+\hat{M} \rho_{i}\right) v_{i}(y, s),(y, s) \in \mathbb{R}^{n} \backslash \pi\left(F_{1}\right) .
\end{array}\right.
$$


Then, $\psi \equiv 0$ on $\mathbb{R}^{n} \backslash Q_{4 R}(X, t)$, and

$$
\mathcal{H}^{n}\left(\pi\left(F_{1}\right)\right) \geq \frac{\epsilon}{2} R^{n+1}, F_{1}:=\Sigma_{\psi} \cap \Delta(X, t, R)
$$

where

$$
\Sigma_{\psi}=\left\{\left(x, x_{n}, t\right) \in \mathbb{R}^{n-1} \times \mathbb{R} \times \mathbb{R}: x_{n}=\psi(x, t)\right\} .
$$

We intend to prove that the function $\psi$ is a regular parabolic Lip $(1,1 / 2)$ function with constants $b_{1}=b_{1}(n, M, \tilde{M}), b_{2}=b_{2}(n, M, \tilde{M})$. By a standard Whitney type argument one can use the fact that $\psi$ is a $\operatorname{Lip}(1,1 / 2)$ function with constant $\hat{M}=\hat{M}(n, M)$, and (3.6)-(3.8), to conclude that (1.3) holds with $b_{1}$ replaced by $c \hat{M}$. To verify this the more delicate case occurs when $(y, s)$ is in the closure of two cubes say $I_{i}, I_{j}$ with $i \in \Lambda, j \notin \Lambda$. However this case follows easily from the fact that $|\hat{\psi}| \leq c \hat{M} R$ and $\left|\partial v_{k} / \partial y_{l}\right|(y, s) \leq c / R$ for $1 \leq l \leq n-1, k=i, j$. Hence it only remains to prove that

$$
\left\|D_{1 / 2}^{t} \psi\right\|_{*} \leq b_{2} \text { for some } b_{2}=b_{2}(n, M, \tilde{M}) .
$$

The proof of (3.11) consists of two step. The first step is to prove that the relevant Carleson norm with respect to $\Sigma_{\psi}$ can be controlled using the local Carleson norm, $\|\nu\|$, defined relative to $\Delta(X, t, 800 R)$. Let $\gamma_{\psi}, \nu_{\psi}$, be as in the statement Definition 2 but with $\Sigma$ replaces by $\Sigma_{\psi}$ as the underlying closed set. Using this notation the first step is to prove that

$$
\left\|\nu_{\psi}\right\|_{+} \leq c(\hat{M})(1+\|\nu\|)
$$

The second step is to prove that the Lip $(1,1 / 2)$ character of $\psi$, and $\left\|\nu_{\psi}\right\|_{+}$, can be used to control $\left\|D_{1 / 2}^{t} \psi\right\|_{*}$ and that, in particular,

$$
\left\|D_{1 / 2}^{t} \psi\right\|_{*} \leq c(\hat{M})(1+\|\nu\|)
$$

To start the proof of (3.12) we note that if $(Y, s) \in \Sigma_{\psi} \cap \overline{Q_{100 R}(X, t)}$, then

$$
d(Y, s, \Sigma) \leq \widetilde{c} \hat{M}^{2} d\left(y, s, \pi\left(F_{1}\right)\right) .
$$

This inequality is trival when $(Y, s) \in F_{1}$. Assume $(Y, s)=(y, s, \hat{\psi}(y, s))$ with $(y, s) \in \bar{I}_{i}$ for some $i$. If $\bar{I}_{i} \cap \bar{I}_{j} \neq \emptyset$ for some $j \notin \Lambda$, then $c \rho_{i} \geq R$ by (3.6), and again (3.14) is trivially satisfied as $(Y, s) \in \Sigma_{\psi} \cap \overline{Q_{100 R}(X, t)}$. Hence, assume that $\bar{I}_{i} \cap \bar{I}_{j}=\emptyset$ for all $j \notin \Lambda$. Then

$$
\begin{aligned}
d((Y, s), \Sigma) & \leq d\left((y, s, \hat{\psi}(y, s)),\left(x_{i}^{\prime}, t_{i}^{\prime}, \hat{\psi}\left(x_{i}^{\prime}, t_{i}^{\prime}\right)\right)\right. \\
& \leq c \rho_{i}+\left|\sum_{i^{\prime} \in \Lambda}\left(\hat{\psi}\left(x_{i^{\prime}}^{\prime}, t_{i^{\prime}}^{\prime}\right)-\hat{\psi}\left(x_{i}^{\prime}, t_{i}^{\prime}\right)+\hat{M} \rho_{i^{\prime}}\right) v_{i^{\prime}}(y, s)\right| \\
& \leq c \hat{M} \rho_{i}
\end{aligned}
$$

by the construction and the $\operatorname{Lip}(1,1 / 2)$ character of $\hat{\psi}$. The proof of $(3.12)$ now follows exactly as in the proof of the corresponding statement in display (2.26) in [HLN] but with $\delta_{1}=1$. In particular, at this stage, having constructed $\psi$ as above and having verified (3.14), the corresponding argument in [HLN] does not use Reifenberg flatness in any decisive step. We omit further details. Finally, for the proof of (3.13), assuming (3.12), we refer to p. 368-373 in [HLN]. This completes our proof of Theorem 1.2. 


\section{Connectivity, Big Pieces, AND An application to Parabolic measure}

Let $\Sigma \subset \mathbb{R}^{n+1}$ be a closed set. Let $\tilde{\gamma}>0$ be given. In the following we let $\Omega \subset \mathbb{R}^{n+1}$ be a domain, i.e., a connected open set, defined as a connected component of $\mathbb{R}^{n+1} \backslash \Sigma$. Assume that $\Sigma \subset \mathbb{R}^{n+1}$ is parabolic ADR in the sense of Definition 1 with constant $M$.

\subsection{Connectivity and big pieces.}

Definition 11. Let $\Sigma$ and $\Omega$ be as above. Let $\epsilon \in(0,1)$ be given. Let $(X, t) \in \partial \Omega$ and $r>0$. We say that $\Omega$ satisfies a local interior chain condition at $(X, t)$, on scale $r$, with constant $\tilde{\gamma}$, if the following is true. Suppose $\left(U_{i}, s_{i}\right) \in \Omega \cap Q_{r}(X, t), i=1,2$, and that $d\left(\left(U_{1}, s_{1}\right), \partial \Omega\right) \geq r / 100, d\left(\left(U_{2}, s_{2}\right), \partial \Omega\right) \geq r / 100$. Then there exists a finite family of cubes $\left\{Q_{r_{i}}\left(X_{i}, t_{i}\right)\right\}_{1}^{l}$, from $\left(U_{1}, s_{1}\right)$ to $\left(U_{2}, s_{2}\right)$, such that

$$
\begin{aligned}
(i) & \left(U_{1}, s_{1}\right) \in Q_{r_{1}}\left(X_{1}, t_{1}\right),\left(U_{2}, s_{2}\right) \in Q_{r_{l}}\left(X_{l}, t_{l}\right), \\
(\text { ii }) & Q_{r_{i+1}}\left(X_{i+1}, t_{i+1}\right) \cap Q_{r_{i}}\left(X_{i}, t_{i}\right) \neq \emptyset \text { for } i=1,2, \ldots, l-1, \\
(\text { iii }) & \left.r_{i}=d\left(X_{i}, t_{i}\right), \partial \Omega\right) / 2, \text { for } i=1,2, \ldots, l, \text { and } l \leq \tilde{\gamma} .
\end{aligned}
$$

Definition 12. Let $\Sigma$ and $\Omega$ be as in Definition 11. Let $\tilde{\gamma}>0$ be given. Let $E \subset \partial \Omega \times(0, \infty)$ be the set of all pairs $((X, t), r),(X, t) \in \partial \Omega$ and $r>0$, for which $\Omega$ does not satisfy a local interior chain condition at $(X, t)$, on scale $r$, with constant $\tilde{\gamma}$. Let

$$
d \tilde{\nu}(Z, \tau, r)=\chi_{E}(Z, \tau, r) d \sigma(Z, \tau) r^{-1} d r,
$$

where $\chi_{E}$ is the indicator function for the set $E$. Given $(Y, s) \in \partial \Omega, R>0$, we say that $\Omega \cap Q_{R}(Y, s)$ satisfies a Carleson chain condition if there exists $\hat{\gamma}$ such that

$$
\tilde{\nu}\left(\left[\partial \Omega \cap Q_{\rho}(X, t)\right] \times(0, \rho)\right) \leq \hat{\gamma} \rho^{n+1},
$$

whenever $(X, t) \in \partial \Omega$ and $Q_{\rho}(X, t) \subset Q_{R}(Y, s)$. The least such $\hat{\gamma}$ in (4.2) is called the Carleson norm of $\left[\partial \Omega \cap Q_{R}(Y, s)\right] \times(0, R)$. We write $\|\tilde{\nu}\|_{+}$for the Carleson norm of $\tilde{\nu}$ when (4.2) holds for all $(X, t) \in \partial \Omega, R, \rho>0$.

Concerning connectivity we prove the following theorem.

Theorem 4.1. Let $\Sigma$ be a closed subset of $\mathbb{R}^{n+1}$ which is parabolic ADR with constant $M$. Assume that $\Sigma$ is parabolic UR with constants $(M, \tilde{M})$. Let $\Omega \subset \mathbb{R}^{n+1}$ be a domain defined as a connected component of $\mathbb{R}^{n+1} \backslash \Sigma$. Assume that $\Omega$ satisfies a uniform Carleson chain condition in the sense of Definition 12 and with constants $\tilde{\gamma}$ and $\hat{\gamma}$. Then $\partial \Omega$ satisfies a uniform interior big pieces of regular parabolic Lip (1,1/2) graphs conditions with constants $\left(\epsilon, b_{1}, b_{2}, C\right)$ for some $\epsilon=\epsilon(n, M, \tilde{M}, \tilde{\gamma}, \hat{\gamma})$, $b_{1}=b_{1}(n, M, \tilde{M}, \tilde{\gamma}, \hat{\gamma}), b_{2}=b_{2}(n, M, \tilde{M}, \tilde{\gamma}, \hat{\gamma})$ and $C=C(n, M, \tilde{M}, \tilde{\gamma}, \hat{\gamma})$.

Proof. To prove Theorem 4.1 we only have to prove that if $\Omega$ is as in the statement of Theorem 4.1, then the additional information that $\Omega$ satisfies a uniform Carleson chain condition, with constants $\tilde{\gamma}$ and $\hat{\gamma}$, in the sense of Definition 12 , implies that $\Omega$ satisfies a synchronized two cube condition in the sense of Definition 9 . To start the proof of this we introduce, for $(Z, \tau) \in \Sigma, r>0$,

$$
\gamma_{\infty}(Z, \tau, r)=\inf _{P} \sup _{(Y, s) \in \Delta(Z, \tau, r)} \frac{d(Y, s, P)}{r},
$$

where the infimum is taken over all $n$ planes $P$ containing a line parallel to the $t$ axis. Given $(Z, \tau), r$ as above it follows, see display (2.2) in [HLN] that

$$
\gamma_{\infty}(Z, \tau, r)^{n+3} \leq 16^{n+3} \gamma(Z, \tau, 2 r) .
$$

In particular, introducing for $\epsilon>0$ the set

$$
F_{\epsilon}=\left\{(Z, \tau, r) \in \Sigma \times(0, R): \gamma_{\infty}(Z, \tau, r)>\epsilon\right\}
$$


we see, by elementary estimates, that

$$
\int_{0}^{\infty} \int_{\Sigma} 1_{F_{\epsilon} \cap\left(Q_{\rho}(X, t) \times(0, \rho)\right)}(Z, \tau, r) d \sigma \frac{d r}{r} \leq c(\epsilon) \tilde{M} \rho^{n+1}
$$

whenever $(X, t) \in \Sigma$ and $0<\rho \leq R$, and where we consider $R$ large and fixed. Consider Definition 12 with constants $\tilde{\gamma}, \hat{\gamma}$. Let $E_{\epsilon} \subset \partial \Omega \times(0, R)$ be the set of all pairs $((X, t), r),(X, t) \in \partial \Omega$ and $0<r \leq R$, for which $\Omega$ does not satisfy a local interior chain condition at $(X, t)$, on scale $\epsilon r$. Then, by assumption, and by the scale invariance of the Carleson measure condition,

$$
\int_{0}^{\infty} \int_{\Sigma} 1_{E_{\epsilon} \cap\left(Q_{\rho}(X, t) \times(0, \rho)\right)}(Z, \tau, r) d \sigma \frac{d r}{r} \leq \hat{\gamma} \rho^{n+1},
$$

whenever $(X, t) \in \Sigma$ and $0<\rho \leq R$. To proceed, consider $(\tilde{Z}, \tilde{\tau}) \in \Sigma$ and $0<$ $\varrho \leq R$. Let $\beta, 0<\beta \ll 1$, be a degree of freedom to be chosen and let $A_{\beta}(\tilde{Z}, \tilde{\tau}, \varrho)$ be the set of all pairs $((Y, s), \rho)$ such that $(Y, s) \in Q_{\varrho / 2}(\tilde{Z}, \tilde{\tau}) \cap \Sigma, \beta \varrho \leq \rho \leq \varrho / 4$, and such that $((Y, s), \rho)$ is in the intersection of the complements of the sets $F_{\epsilon}$ and $E_{\epsilon}$. Assume that $A_{\beta}(\tilde{Z}, \tilde{\tau}, \varrho)$ is empty. This implies that if $(Y, s) \in Q_{\varrho / 2}(\tilde{Z}, \tilde{\tau}) \cap \Sigma$, $\beta \varrho \leq \rho \leq \varrho / 4$, then $((Y, s), \rho) \in F_{\epsilon} \cup E_{\epsilon}$. Hence

$$
\begin{aligned}
(c(\epsilon) \tilde{M}+\hat{\gamma}) \varrho^{n+1} & \geq \int_{0}^{\infty} \int_{\Sigma} 1_{F_{\epsilon} \cup E_{\epsilon} \cap\left(Q_{\varrho / 2}(\tilde{Z}, \tilde{\tau}) \times(0, \varrho)\right)}(Z, \tau, r) d \sigma \frac{d r}{r} \\
& \geq c^{-1} \varrho^{n+1} \int_{\beta \varrho}^{\varrho / 4} \frac{d r}{r}=c^{-1} \varrho^{n+1} \log (1 /(2 \beta)) .
\end{aligned}
$$

We can conclude that if we in this construction choose $\beta=\beta(n, M, \tilde{M}, \epsilon, \tilde{\gamma}, \hat{\gamma})$ large enough, then the set $A_{\beta}(\tilde{Z}, \tilde{\tau}, \varrho)$ is non-empty whenever $(\tilde{Z}, \tilde{\tau}) \in \Sigma$ and $0<\varrho \leq R$. Hence, consider $((Y, s), \rho) \in A_{\beta}(\tilde{Z}, \tilde{\tau}, \varrho)$. Then by construction, and the definition of the set $F_{\epsilon}$, there exists a time-independent plane $P$ such that

$$
d((\tilde{Y}, \tilde{s}), P) \leq \epsilon \rho \text { whenever }(\tilde{Y}, \tilde{s}) \in \Delta(Y, s, \rho) .
$$

Let $\left(n_{P}, 0\right)$ denote the normal to the plane $P$ and set $\left(Y_{1}, s_{1}\right)=\left(Y+10 \epsilon \rho n_{P}, s\right)$, $\left(Y_{2}, s_{2}\right)=\left(Y-10 \epsilon \rho n_{P}, s\right)$. Using (4.9) we see that $\left(Y_{1}, s_{1}\right),\left(Y_{2}, s_{2}\right) \in Q_{1000 \epsilon}(Y, s)$. Furthermore, to connect $\left(Y_{1}, s_{1}\right)$ and $\left(Y_{2}, s_{2}\right)$ by a sequence of cubes as in Definition 12 we see that the number of cubes needed is at least on the magnitude of $\log \left(\epsilon^{-1}\right)$. However, by construction $\Omega$ satisfies a local interior chain condition at $(Y, s)$, on scale $\epsilon \rho$, with constant $\tilde{\gamma}$. Hence, if $\left(Y_{1}, s_{1}\right),\left(Y_{2}, s_{2}\right)$ would be in the same component these two statements would contradict each other. Hence $\left(Y_{1}, s_{1}\right),\left(Y_{2}, s_{2}\right)$ must be in different components and we can conclude that $\Omega$ satisfies a synchronized two cube condition for some constant $\gamma_{0}(n, M, \tilde{M}, \tilde{\gamma}, \hat{\gamma})$. This completes the proof of Theorem 4.1 .

4.2. An application to parabolic measure. Let $\Sigma$ and $\Omega$ be as above. We are interested in the bounded continuous Dirichlet problem and the parabolic measure in $\Omega$. To start our outline, we recall the notion of thermal capacity introduced in [EG]. Let $K$ be a closed subset of $\mathbb{R}^{n+1}$ and let $\mathcal{M}^{+}(K)$ be the collection of all non-negative Radon measures on $\mathbb{R}^{n+1}$ with support contained in $K$. We define, following [EG], the thermal capacity of $K$ as

$$
\operatorname{Cap}(K)=\sup \left\{\mu\left(\mathbb{R}^{n+1}\right): \mu \in \mathcal{M}^{+}(K), P_{\mu} \leq 1 \text { on } \mathbb{R}^{n+1}\right\}
$$

where

$$
P_{\mu}(X, t)=\int_{\mathbb{R}^{n+1}} W(X-Y, t-s) d \mu(Y, s),
$$


and where $W$ is the standard heat kernel on $\mathbb{R}^{n+1}$. Let $(X, t) \in \partial \Omega$ and consider, for $k \in \mathbb{Z}_{+}$, the sets

$$
A\left(X, t, 2^{-k}\right)=\left\{(Y, s) \in \mathbb{R}^{n+1}:\left(4 \pi 2^{-k}\right)^{-n / 2} \leq W(X-Y, t-s) \leq\left(2 \pi 2^{-k}\right)^{-n / 2}\right\} .
$$

Consider the set $\partial \Omega \cap A\left(X, t, 2^{-k}\right)$ and let

$$
\mu_{k}(E)=\sigma(E \cap \partial \Omega) /\left(2^{-k n / 2} \sigma\left(\partial \Omega \cap A\left(X, t, 2^{-k}\right)\right)\right),
$$

whenever $E \subset \mathbb{R}^{n+1}$ is a Borel set. It is then easy to see, using that $\Sigma$ is parabolic ADR, that $P_{\mu_{k}} \leq c$ on $\mathbb{R}^{n+1}$, for some $c$ independent of $k$. Hence,

$$
\operatorname{Cap}\left(\partial \Omega \cap A\left(X, t, 2^{-k}\right)\right) \geq c^{-1} 2^{-k n / 2},
$$

and using the Wiener criterium in $[\mathrm{EG}]$ we can conclude that any point $(X, t) \in \partial \Omega$ is regular for the bounded continuous Dirichlet problem for the heat equation in $\Omega$. By analogy, the same also holds for the adjoint heat equation in $\Omega$. Using this, exhausting $\Omega$ by sets of the form $\Omega \cap Q_{R}(0,0)$, letting $R \rightarrow \infty$, and applying Perron-Wiener-Brelot type arguments we can conclude that the bounded continuous Dirichlet problems for the heat equation, adjoint heat equation, in $\Omega$ always have unique solutions. Given $(\hat{X}, \hat{t}) \in \Omega$ we let $\omega(\hat{X}, \hat{t}, \cdot)$ be the caloric measure, at $(\hat{X}, \hat{t}) \in \Omega$, associated to the heat equation in $\Omega$. To proceed we will now assume that $\Omega$ has additional connectivity properties.

Definition 13. Let $\Sigma$ and $\Omega$ be as in Definition 11. We say that $\Omega$ has the Harnack chain property if the following condition is satisfied for some $\gamma \geq 100$. Suppose $\left(U_{i}, s_{i}\right) \in \Omega, i=1,2$, with $\left(s_{2}-s_{1}\right)^{\frac{1}{2}}>\gamma^{-1} d\left(\left(U_{1}, s_{1}\right),\left(U_{2}, s_{2}\right)\right)$. Then there exists a Harnack chain $\left\{Q_{r_{i}}\left(X_{i}, t_{i}\right)\right\}_{1}^{l}$ from $\left(U_{1}, s_{1}\right)$ to $\left(U_{2}, s_{2}\right)$ such that the following hold for some $c(\gamma) \geq 1$.

$$
\begin{aligned}
(i) & \left(U_{1}, s_{1}\right) \in Q_{r_{1}}\left(X_{1}, t_{1}\right),\left(U_{2}, s_{2}\right) \in Q_{r_{l}}\left(X_{l}, t_{l}\right), \\
(i i) & Q_{r_{i+1}}\left(X_{i+1}, t_{i+1}\right) \cap Q_{r_{i}}\left(X_{i}, t_{i}\right) \neq \emptyset \text { for } i=1,2, \ldots, l-1, \\
(i i i) & c(\gamma)^{-1} d\left(\left(X_{i}, t_{i}\right), \partial \Omega\right) \leq r_{i} \leq c(\gamma) d\left(\left(X_{i}, t_{i}\right), \partial \Omega\right), \text { for } i=1,2, \ldots, l, \\
(\text { iv }) & t_{i+1}-t_{i} \geq c(\gamma)^{-1} r_{i}^{2}, \text { for } i=1,2, \ldots, l, \\
(v) & l \leq c(\gamma) \log \left(2+\frac{d\left(\left(U_{1}, s_{1}\right),\left(U_{2}, s_{2}\right)\right)}{\min \left[d\left(\left(U_{1}, s_{1}\right), \partial \Omega\right), d\left(\left(U_{2}, s_{2}\right), \partial \Omega\right)\right]}\right) .
\end{aligned}
$$

Remark 4.1. Note that the connectivity conditions in Definition 12 and Definition 13 are different. The condition in Definition 12 is a condition concerning the (local) connectivity of $\Omega$ formulated in terms of a Carleson measure condition. The condition in Definition 13 is constructed in order to be able to use the Harnack inequality for the heat equation in an efficient way to compare the values of non-negative solutions respecting the time-lag in the parabolic Harnack inequality. Definition 12 makes no reference to Harnack chains.

Assume in the following that $\partial \Omega$ satisfies a synchronized two cube condition in the sense of Definition 8. For $(X, t), r>0$, and $A \geq 100$, we define

$$
\Gamma_{A}^{+}(X, t, r)=\left\{(Y, s):|Y-X|^{2} \leq A|s-t|,|s-t| \geq 5 r^{2}, s>t\right\}
$$

Definition 14. Let $(X, t) \in \partial \Omega, r>0$, and consider $(\hat{X}, \hat{t}) \in \Omega \cap \Gamma_{A}^{+}(X, t, 4 r)$. We say that $\omega(\cdot)=\omega(\hat{X}, \hat{t}, \cdot)$ satisfies the $A_{\infty}$ condition on $\partial \Omega \cap Q_{r}(X, t)$, with constants $L$ and $\lambda>0$ if the following is true. $d \omega / d \sigma=h$ exists on $\partial \Omega \cap Q_{r}(X, t)$ and

$$
\int_{\partial \Omega \cap Q_{\rho}(\tilde{X}, \tilde{t})} h^{1+\lambda} d \sigma \leq L \sigma\left(Q_{\rho}(\tilde{X}, \tilde{t})\right)^{-\lambda}\left(\omega\left(Q_{\rho}(\tilde{X}, \tilde{t})\right)\right)^{1+\lambda}
$$

whenever $(\tilde{X}, \tilde{t}) \in \partial \Omega, Q_{2 \rho}(\tilde{X}, \tilde{t}) \subset Q_{r}(X, t)$. 
Theorem 4.2. Let $\Sigma$ be a closed subset of $\mathbb{R}^{n+1}$ which is parabolic ADR with constant $M$. Assume that $\Sigma$ is parabolic UR with constants $(M, \tilde{M})$. Let $\Omega \subset \mathbb{R}^{n+1}$ be a domain defined as a connected component of $\mathbb{R}^{n+1} \backslash \Sigma$. Assume that $\Omega$ satisfies a synchronized two cube condition with constant $\gamma_{0}$ in the sense of Definition 9. Assume that $\Omega$ has the Harnack chain property in the sense of Definition 13 and with constant $\gamma$. Let $(X, t) \in \partial \Omega, r>0, A \geq 100$, and consider $(\hat{X}, \hat{t}) \in \Omega \cap \Gamma_{A}^{+}(X, t, 4 r)$. Then $\omega(\hat{X}, \hat{t}, \cdot)$ is a doubling measure in the sense that there exists a constant $c=$ $c\left(n, M, \gamma_{0}, \gamma, A\right)$ such that

$$
\omega\left(\hat{X}, \hat{t}, \partial \Omega \cap Q_{2 \rho}(\tilde{X}, \tilde{t})\right) \leq c \omega\left(\hat{X}, \hat{t}, \partial \Omega \cap Q_{\rho}(\tilde{X}, \tilde{t})\right),
$$

whenever $(\tilde{X}, \tilde{t}) \in \partial \Omega, Q_{\rho}(\tilde{X}, \tilde{t}) \subset Q_{2 r}(X, t)$. Furthermore, $\omega(\hat{X}, \hat{t}, \cdot)$ satisfies the $A_{\infty}$ condition on $\partial \Omega \cap Q_{r}(X, t)$ in the sense of Definition 14 with constants $L=$ $L\left(n, M, \tilde{M}, \gamma_{0}, \gamma, A\right)$ and $\lambda\left(n, M, \tilde{M}, \gamma_{0}, \gamma, A\right)>0$.

Proof. If $\Omega$ satisfies a synchronized two cube condition in the sense of Definition 9, and if $\Omega$ has the Harnack chain property in the sense of Definition 13 with constant $\gamma$, then $\Omega$ is a parabolic NTA-domain in the sense of [LM] and [HLN1]. Therefore, we see that (4.12) follows from [LM] and [HLN1]. To prove the statement that $\omega(\hat{X}, \hat{t}, \cdot)$ satisfies the $A_{\infty}$ condition on $\partial \Omega \cap Q_{r}(X, t)$ in the sense of Definition 14 , we first observe that the assumptions stated in the theorem imply, see Theorem 1.2 , that $\Omega$ contains uniform interior big pieces of regular parabolic $\operatorname{Lip}(1,1 / 2)$ graphs. Using this, and that $\Omega$ is a parabolic NTA-domain, we can follow the proof of Theorem 1 in [HLN1] essentially verbatim to conclude that $\omega(\hat{X}, \hat{t}, \cdot)$ satisfies the $A_{\infty}$ condition on $\partial \Omega \cap Q_{r}(X, t)$. At this stage in [HLN], having established uniform interior big pieces of regular parabolic Lip $(1,1 / 2)$ graphs, the assumption concerning Reifenberg flatness imposed in [HLN] is only used to ensure that $\Omega$ is a parabolic NTA-domain, something we in our setting already have concluded. We therefore omit further details of the proof and simply refer to the proof of Theorem 1 in [HLN1].

Acknowledgement. The authors thank an anonymous referee for a careful reading of the paper and for valuable suggestions.

\section{REFERENCES}

[BL] B. Bennewitz and J.L. Lewis, On weak reverse Hölder inequalities for nondoubling harmonic measures, Complex Var. Theory Appl. 49 (2004), no. 7-9, 571-582.

[DJ] G. David, D. Jerison, Lipschitz Approximation to Hypersurfaces, Harmonic Measure, and Singular Integrals, Indiana University Mathematics Journal 39 (1990), 831-845.

[DS] G. David and S. Semmes, Singular integrals and rectifiable sets in $\mathbb{R}^{n}$ : Au-dela des graphes lipschitziens, Asterisque 193 (1991).

[DS1] G. David and S. Semmes, Analysis of and on Uniformly Rectifiable Sets, Mathematical Monographs and Surveys 38, AMS 1993.

[EG] Evans and Gariepy, Wieners test for the heat equation. Arch. Rational Mech. Anal. 78 (1982), 293-314.

[H] S.Hofmann, Parabolic singular integrals of Calderón type, rough operators, and caloric layer potentials, Duke Math. J. 90 (1997), 209-259.

[HL] S. Hofmann and J. Lewis, Solvability and representation by caloric layer potentials in time-varying domains, Ann. of Math. 144 (1996), 349-420.

[HL1] S. Hofmann and J. Lewis, The Dirichlet problem for parabolic operators with singular drift terms, Mem. Amer. Math. Soc. 151 (2001).

[HLN] S. Hofmann, J. Lewis and K. Nyström, Existence of big pieces of graphs for parabolic problems, Annales Acad. Sci. Fenn. Mathematica 28 (2003), 355-384.

[HLN1] S. Hofmann, J. Lewis and K. Nyström, Caloric measure in parabolic flat domains, Duke math. J 122 (2004), 281-345. 
[JK] D. Jerison and C. Kenig, Boundary behaviour of harmonic functions in nontangentially accessible domains, Advances in Math. 46 (1982), 80-147.

[LM] J. Lewis and M. Murray, The method of layer potentials for the heat equation in timevarying domains, Mem. Amer. Math. Soc. 114 (1995).

[LS] J. Lewis and J. Silver, Parabolic measure and the Dirichlet problem for the heat equation in two dimensions, Indiana Univ. Math. J. 37 (1988), 801-839.

[LV] J. L. Lewis and A. Vogel, Uniqueness in a free boundary problem, Communications in PDE, 31 (2006), 1591-1614.

[LV1] J. L. Lewis and A. Vogel, Symmetry theorems and uniform rectifiability, Boundary Value Problems Vol. 2007 (2007), article ID 030190, 59 pages.

[NS] K. Nyström and M. Strömqvist, Analysis of and on parabolic uniformly rectifiable sets satisfying a synchronized two cube condition, in preparation.

[RN1] J. Rivera-Noriega, A parabolic version of Corona decompositions. Illinois math journal 53 (2009), 533-559.

[RN3] J. Rivera-Noriega, Two results over sets with big pieces of parabolic Lipschitz graphs. Houston Journal of Mathematics 36 (2010) 619-635.

[RN3] J. Rivera-Noriega, Parabolic singular integrals and uniform rectifiable sets in the parabolic sense, J. Geometrical analysis 23 (2013), 1140-1157.

Kaj Nyström, Department of Mathematics, Uppsala University, S-751 06 Uppsala, SWEDEN

E-mail address: kaj.nystrom@math.uu.se

Martin Strömqvist, Department of Mathematics, Uppsala University, S-751 06 UppSALA, SWEDEN

E-mail address: martin.stromqvist@math.uu.se 